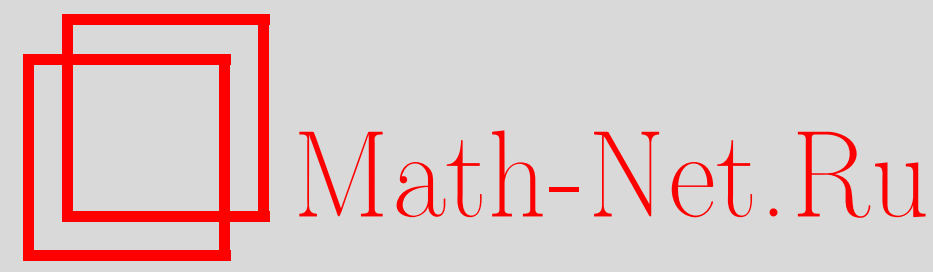

И. В. Артамкин, Производящие функции модулярных графов и уравнение Бюргерса, Матем. сб., 2005, том 196, номер 12, 3-32

DOI: https://doi.org/10.4213/sm1441

Использование Общероссийского математического портала Math-Net.Ru подразумевает, что вы прочитали и согласны с пользовательским соглашением http://www . mathnet.ru/rus/agreement

Параметры загрузки:

IP: 3.89 .197 .203

26 апреля 2023 г., 13:14:59 
УДК 512.772

\section{И.В. Артамкин}

\section{Производящие функции модулярных графов и уравнение Бюргерса}

Доказьвается, что производящие функции модулярных графов удовлетворяют уравнению Бюргерса, что позволяет единообразно получать производящие функции для виртуальной эйлеровой характеристики и многочлена Пуанкаре многообразия модулей пунктированных кривых $\bar{M}_{g, n}$, а также для количества (с весами $1 / \mid$ Aut $G \mid$ ) модулярных графоов $G$ определенного вида.

Библиография: 10 названий.

\section{§1. Введение}

Алгебраическая кривая, имеющая не более чем простейшие двойные особые точки, на которой отмечено $n \geqslant 0$ упорядоченных неособых точек, называется стабильной по Делиню-Мамфорду пунктированной кривой. При этом стабильность означает, что каждая неприводимая рациональная компонента такой кривой имеет по крайней мере три особые и/или отмеченные точки, а эллиптическая компонента содержит по крайней мере одну такую точку. Двойственный модулярньй граф̆ пунктированной кривой $X$ - это граф Г, вершинами которого является множество неприводимых компонент кривой $X$, ребрами - множество двойных особых точек, а полуребрами - множество отмеченных точек. Для каждого модулярного графа $Г$ рассмотрим пространство модулей $M_{\Gamma}$ кривых, для которых $\Gamma$ является двойственным модулярным графом. Тогда компактификация Делиня-Мамфорда пространства модулей $M_{g, n}$ алгебраических кривых рода $g$ с отмеченными $n$ упорядоченными точками имеет стратификацию

$$
\bar{M}_{g, n}=\underset{\substack{\text { по всем модулярным графам } \Gamma \\ \text { рода } g \text { с } n \text { полуребрами }}}{\bigcup} M_{\Gamma} \text {. }
$$

Формально модулярный граф Г можно определить следующим набором данных: $\left(V, \vec{E}, i, \vec{E}_{-}, s, g, l\right)$, где

1) $V$ - конечное множество вериин $\Gamma$;

2) $\vec{E}$ - конечное множество ориентированных ребер модулярного графа $\Gamma$;

3) $i: \vec{E} \rightarrow \vec{E}-$ инволющия, меняющая ориентацию (без неподвижных точек);

4) $\overrightarrow{E_{-}}-$множество исходящих ориентированных ребер модулярногографа Г; требуется, чтобы $\vec{E}=\overrightarrow{E_{-}} \cup i\left(\overrightarrow{E_{-}}\right)$, так что каждое ребро является либо входящим, либо исходящим; 
5) $s: \overrightarrow{E_{-}} \rightarrow V$ - сюръективное отображение, ставящее в соответствие каждому исходящему ребру из $\overrightarrow{E_{-}}$вершину, из которой оно выходит;

6) $g: V \rightarrow\{0,1,2,3, \ldots\}$ - функция, задающая род вершины;

7) множество $\vec{H}_{-}=\vec{E}_{-} \backslash i\left(\vec{E}_{-}\right)$назьвается множеством исходящих полуребер (т.е. ребер, инцидентных только одной вершине), для непустого $\vec{H}_{-}$ биекция $l: \vec{H}_{-} \stackrel{\sim}{\rightarrow}\{1,2,3, \ldots, n\}$ определяет упорядочение множества полуребер модулярного графа $Г$.

Множество неориентированных ребер модулярного графа Г - это фактормножество $E=(\vec{E} \cap i(\vec{E})) / i$. Изоморфизм двух модулярных графов задается парой биекций между множествами вершин и множествами ориентированных ребер, сохраняющих все описанные выше данные; заметим, что нетривиальный автоморфизм модулярного графа может быть тождественньм как на множестве вершин, так и на множестве неориентированных ребер. Количество исходящих из вершины $v$ ориентированных ребер $\nu(v)=\left|s^{-1}(v)\right|$ называется валентностью вериины $v \in V(\Gamma)$. Модулярный граф̆ называется стабильнылм, если $2(g(v)-1)+\nu(v)>0$ для любой вершины $v \in V(\Gamma)$. Число $g(\Gamma)=\sum_{v \in V(\Gamma)} g(v)+\operatorname{dim} H_{1}(\Gamma)-p o д$ связного модулярного графа $\Gamma ; n(\Gamma)$ - число полуребер модулярного графа Г. Предположим, что $g(v)=0$ для всех вершин модулярного графа $\Gamma$, такие модулярные графы в дальнейшем будем называть комбинаторными графами или просто графами. В этом случае стабильность означает, что валентность каждой вершины не меньше трех. В этой работе мы будем рассматривать только связные стабильные модулярные графы.

Пусть $\left\{\mu_{g, n}, 2(g-1)+n>0\right\}-$ множество (коммутативных) переменных, $\Gamma-$ модулярный граф. Определим моном

$$
\mu(\Gamma)=\frac{1}{|\operatorname{Aut} \Gamma|} \prod_{v \in V(\Gamma)} \mu_{g(v), \nu(v)}
$$

где Aut $\Gamma$ - группа автоморфизмов модулярного графа Г. Пусть $\mathscr{G}_{g, n}^{k}-$ множество всех модулярных графов рода $g$ с $k$ ребрами и $n$ полуребрами, рассмотрим многочлены

$$
\mu_{g, n}^{k}=\sum_{\Gamma \in \mathscr{G}_{g, n}^{k}} \mu(\Gamma)
$$

и производящие функции

$$
\Psi(s, t, \hbar)=\sum_{g=0}^{\infty} \sum_{n=0}^{\infty} \sum_{k=0}^{3 g-3+n} \mu_{g, n}^{k} \frac{t^{n}}{n !} s^{k} \hbar^{g-1}
$$

и

$$
\Phi(s, t, \hbar)=\frac{\partial \Psi(s, t, \hbar)}{\partial t}=\sum_{g=0}^{\infty} \sum_{n=1}^{\infty} \sum_{k=0}^{3 g-3+n} \mu_{g, n}^{k} \frac{t^{n-1}}{(n-1) !} s^{k} \hbar^{g-1}
$$


Заметим, что

$$
\Psi(1, t, \hbar)=\sum_{g=0}^{\infty} \sum_{n=0}^{\infty} \sum_{\Gamma \in \mathscr{G}_{g, n}} \mu(\Gamma) \frac{t^{n}}{n !} \hbar^{g-1}
$$

- статистическая сумма, обычно рассматриваемая в квантовой теории поля (здесь $\mathscr{G}_{g, n}=\bigcup_{k} \mathscr{G}_{g, n}^{k}-$ множество всех модулярных графов рода $g$ с $n$ полуребрами).

Доказывается, что функции $\Phi$ и $\Psi$ удовлетворяют уравнению Бюргерса.

ТЕОРема 1.1. Функиия $\Psi(s, t, \hbar)$ удовлетворяет потенииальной форме уравнения Бюргерса

$$
\frac{\partial \Psi}{\partial s}=\frac{\hbar}{2}\left[\frac{\partial^{2} \Psi}{\partial t^{2}}+\left(\frac{\partial \Psi}{\partial t}\right)^{2}\right]
$$

а функиия $\Phi(s, t, \hbar)$ удовлетворяет уравнению Бюргерса

$$
\frac{\partial \Phi}{\partial s}=\frac{\hbar}{2}\left[\frac{\partial^{2} \Phi}{\partial t^{2}}+2 \Phi \frac{\partial \Phi}{\partial t}\right] .
$$

Заметим, что начальное условие $\Psi(0, t, \hbar)$ для уравнения Бюргерса - это сумма по множеству $\mathscr{G}_{g, n}^{0}$ модулярных графов, не имеющих ребер. Для каждой пары $(g, n)$ такой, что $2(g-1)+n>0$, множество $\mathscr{G}_{g, n}^{0}$ состоит только из одного модулярного дерева $S_{g, n}$, имеюшего одну вершину рода $g$ и $n$ полуребер, которое соответствует пространству модулей неособых пунктированных кривых: $M_{S_{g, n}}=M_{g, n}$. Поэтому

$$
\Psi(0, t, \hbar)=\sum_{\substack{g \geqslant 0, n \geqslant 0 \\ 2(g-1)+n>0}} \mu_{g, n} \frac{t^{n}}{n !} \hbar^{g-1} .
$$

Можно несколькими способами придавать значения переменным $\left\{\mu_{g, n}\right\}$ так, чтобы получались интересные производящие функции $\Psi$ (или $\Phi$ ).

\section{1. Считающие функции для комбинаторных графов определенного} типа.

а) Возьмем целое $d \geqslant 3$ и положим

$$
\mu_{g, n}= \begin{cases}1, & \text { если } g=0 \text { и } n=d ; \\ 0 & \text { в остальных случаях. }\end{cases}
$$

Тогда $\Psi$ является считаюшей функцией для $d$-валентных (комбинаторных) графов:

$$
\Psi(s, t, \hbar)=\sum_{g, n, k}\left[\sum_{\substack{\text { подала } g \text { с } k \text { ребрами } \\ \text { и } n \text { полуребрами }}} \frac{1}{|\mathrm{Aut} \Gamma|}\right] \frac{t^{n}}{n !} s^{k} \hbar^{g-1} .
$$

(Заметим, что сумма в скобках ненулевая только при $(d-2) k=n+d(g-1)$.)

В этом случае начальные условия выглядят так:

$$
\Psi(0, t, \hbar)=\frac{t^{d}}{d ! \hbar} \quad \text { или } \quad \Phi(0, t, \hbar)=\frac{t^{d-1}}{(d-1) ! \hbar} .
$$


Ниже мы приводим явные формулы решения для наиболее интересного случая трехвалентных графов $(d=3)$.

б) Положим

$$
\mu_{g, n}= \begin{cases}1, & \text { если } g=0 ; \\ 0 & \text { в остальных случаях. }\end{cases}
$$

Получим считаюшую функцию для всех стабильных (комбинаторных) графов:

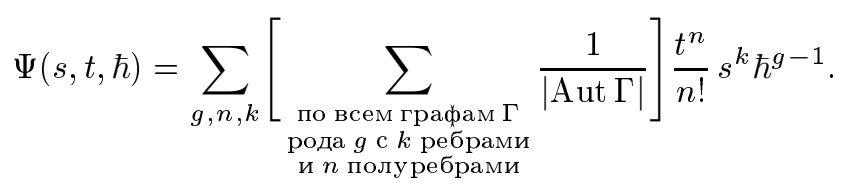

Начальным условием в этом случае будет

$$
\Psi(0, t, \hbar)=\left(e^{t}-\frac{t^{2}}{2}-t-1\right) \frac{1}{\hbar} .
$$

в) Замена $\mu_{g, n}=1$ для всех $g, n$ дает считающую функцию для всех модулярных графов:

$$
\Psi(s, t, \hbar)=\sum_{g, n, k}\left[\sum_{\substack{\text { по всем модулярным графам } \\ \text { рода } g \text { с } k \text { peбрами } \\ \text { и } n \text { полуребрами }}} \frac{1}{|\mathrm{Aut} \Gamma|}\right] \frac{t^{n}}{n !} s^{k} \hbar^{g-1} .
$$

Начальное условие в этом случае:

$$
\Psi(0, t, \hbar)=\left(e^{t}-\frac{t^{2}}{2}-t-1\right) \frac{1}{\hbar}-1+\frac{e^{t}}{1-\hbar} .
$$

1.2. Виртуальная мотивная мера $M_{g, n}$. Выберем некоторую "мотивную меру" $v$, сопоставляюшую каждому неособому алгебраическому многообразию $X$ элемент $v(X)$ некоторой коммутативной $\mathbb{Q}$-алгебры, удовлетворяющий следующим условиям:

a) $v(X \backslash Y)+v(Y)=v(X)$ для любого замкнутого неособого подмногообразия $Y \subset X$

b) $v(X \times Z)=v(X) v(Z)$.

Соответствуюшая "виртуальная мотивная мера" $\widetilde{v}$ орбиобразия $X$ может быть задана как $\widetilde{v}(X)=v(\widetilde{X}) / N$, где $\widetilde{X} \rightarrow X$ - неразветвленное накрытие орбиобразий степени $N$, а $X$ неособо. (Достаточно иметь такое накрытие для каждого страта некоторой стратификации $X$.) Положим $\mu_{g, n}=\widetilde{v}\left(M_{g, n}\right)$. Тогда нетрудно вывести, что

$$
\mu(\Gamma)=\widetilde{v}\left(M_{\Gamma}\right)
$$

и

$$
\mu_{g, n}^{k}=\widetilde{v}\left(M_{g, n}^{k}\right)
$$


где $M_{g, n}^{k}$-пространство модулей стабильных по Делиню-Мамфорду $n$-пунктированных кривых рода $g$, имеющих ровно $k$ особых точек. Заметим, что при заданных $g$ и $n$ пространства $M_{g, n}^{k}$ образуют стратификацию $\bar{M}_{g, n}$ и $\operatorname{codim}_{\bar{M}_{g, n}} M_{g, n}^{k}=k$. Таким образом, производящие функции (1.4) имеют вид

$$
\Psi(s, t, \hbar)=\sum_{g=0}^{\infty} \sum_{n=0}^{\infty} \sum_{k=0}^{3 g-3+n} \widetilde{v}\left(M_{g, n}^{k}\right) \frac{t^{n}}{n !} s^{k} \hbar^{g-1}
$$

а статистическая сумма (1.6) становится производящей функцией для величин $\widetilde{v}\left(\bar{M}_{g, n}\right)$ для компактифицированного пространства модулей $\bar{M}_{g, n}$ :

$$
\Psi(1, t, \hbar)=\sum_{g=0}^{\infty} \sum_{n=0}^{\infty} \widetilde{v}\left(\bar{M}_{g, n}\right) \frac{t^{n}}{n !} \hbar^{g-1} .
$$

Начальное условие (1.9) становится производящей функцией для величин $\widetilde{v}\left(M_{g, n}\right)$ для пространства модулей $M_{g, n}$ неособых кривых:

$$
\Psi(0, t, \hbar)=\sum_{g=0}^{\infty} \sum_{n=0}^{\infty} \widetilde{v}\left(M_{g, n}\right) \frac{t^{n}}{n !} \hbar^{g-1} .
$$

В качестве такой “виртуальной мотивной меры” $\widetilde{v}$ можно взять виртуальный многочлен Пуанкаре от $X$ (см. [1] или [2]), или виртуальную эйлерову характеристику $X$, или число точек $X\left(\mathbb{F}_{q}\right)$ над конечным полем $\mathbb{F}_{q}$. Но явная формула для начального условия известна только для виртуальной эйлеровой характеристики. Она дается известным результатом Харера-Загира [3]: для $g>0$

$$
\widetilde{\chi}\left(M_{g, n}\right)=(-1)^{n} \frac{(2 g-3+n) !(2 g-1)}{(2 g) !} B_{2 g}
$$

для $g \geqslant 2, n \geqslant 0$ или $g=1, n \geqslant 1$. Объединив это со случаем $g=0$ (см. [2] или $\S 6)$, получаем производящие функции

$$
\begin{aligned}
\Psi(0, t, \hbar)= & \frac{2(1+t)^{2} \ln (1+t)-2 t-3 t^{2}}{4 \hbar}-\frac{B_{2}}{2} \ln (1+t) \\
& +\sum_{g=2}^{\infty} \frac{B_{2 g}}{2 g(2 g-2)} \frac{\hbar^{g-1}}{(1+t)^{2 g-2}}
\end{aligned}
$$

и

$$
\Phi(0, t, \hbar)=\frac{(1+t) \ln (1+t)-t}{\hbar}-\sum_{g=1}^{\infty} \frac{B_{2 g}}{2 g} \frac{\hbar^{g-1}}{(1+t)^{2 g-1}} .
$$

Во всех описанных случаях нам нужно решить задачу Коши для уравнения Бюргерса с начальньм условием, заданным одной из формул $(1.12),(1.15),(1.17)$ или (1.25). Уравнение (1.7) можно линеаризовать преобразованием Коула-Хопфа (cм. [4], [5]):

$$
\Psi=\ln F .
$$


Подставляя в (1.7), получаем уравнение теплопроводности

$$
\frac{\partial F}{\partial s}=\frac{\hbar}{2} \frac{\partial^{2} F}{\partial t^{2}}
$$

с начальным условием

$$
F(0, t, \hbar)=e^{\Psi(0, t, \hbar)}=e^{\sum_{g=0}^{\infty} \sum_{n=0}^{\infty} \mu_{g, n} \frac{t^{n}}{n !} \hbar^{g-1}}
$$

Решение задачи Коши для (1.27) с начальным условием (1.28), формально выраженное через интеграл Пуассона, для $s=1$ известно как теорема Вика (см. [6]):

$$
\Psi(s, t, \hbar)=\ln \left(\frac{1}{\sqrt{2 \pi \hbar s}} \int_{-\infty}^{\infty} e^{\Psi(0, \xi, \hbar)-\frac{(t-\xi)^{2}}{2 s \hbar}} d \xi\right) .
$$

Конечно, (1.29) должно рассматриваться как равенство формальных рядов Лорана от $\hbar$. К сожалению, во всех описанных случаях применение интеграла Пуассона неправомерно, так как начальные условия $(1.12),(1.15),(1.17)$ и (1.25) не ограничены, так что несобственный интеграл (1.29) расходится. Более того, А.Н. Тихонов в 1935 г. (см. [7]) доказал, что решение задачи Коши для уравнения теплопроводности с начальным условием, растущим быстрее $e^{t^{2}}$, уже не единственно. А это как раз имеет место во всех рассматриваемых нами случаях. Например, для считающей функции для трехвалентных графов имеем следующий интеграл:

$$
\Psi(s, t, \hbar)=\ln \left(\frac{1}{\sqrt{2 \pi \hbar s}} \int_{-\infty}^{\infty} e^{\frac{1}{\hbar}\left[\frac{\xi^{3}}{6}-\frac{(t-\xi)^{2}}{2 s}\right]} d \xi\right)
$$

В (1.57) приводится явная формула для однопараметрического семейства решений (1.27) с начальньм условием $e^{t^{3} /(6 \hbar)}$.

Конечно, можно рассматривать (1.29) как распределение, но это вряд ли может помочь найти коэффициенты производящей функции. Вместо этого можно разложить решение по степеням $\hbar$ :

$$
\begin{aligned}
& \Phi(s, t, \hbar)=\sum_{g=0}^{\infty} \Phi_{g}(s, t) \hbar^{g-1}, \\
& \Psi(s, t, \hbar)=\sum_{g=0}^{\infty} \Psi_{g}(s, t) \hbar^{g-1},
\end{aligned}
$$

где $\Phi_{g}=\partial \Psi_{g} / \partial t$, и попытаться найти рекуррентную формулу для функций $\Phi_{g}$ или $\Psi_{g}$.

Тогда получаем квазилинейное уравнение для $\Phi_{0}$

$$
\frac{\partial \Phi_{0}}{\partial s}=\Phi_{0} \frac{\partial \Phi_{0}}{\partial t}
$$


и рекуррентные квазилинейные уравнения для $\Phi_{g}$ и $\Psi_{g}$ при $g>0$

$$
\begin{aligned}
& \frac{\partial \Phi_{g}}{\partial s}=\frac{1}{2} \frac{\partial^{2} \Phi_{g-1}}{\partial t^{2}}+\Phi_{0} \frac{\partial \Phi_{g}}{\partial t}+\Phi_{g} \frac{\partial \Phi_{0}}{\partial t}+\sum_{i=1}^{g-1} \Phi_{i} \frac{\partial \Phi_{g-i}}{\partial t} \\
& \frac{\partial \Psi_{g}}{\partial s}=\frac{1}{2} \frac{\partial^{2} \Psi_{g-1}}{\partial t^{2}}+\Phi_{0} \frac{\partial \Psi_{g}}{\partial t}+\frac{1}{2} \sum_{i=1}^{g-1} \frac{\partial \Psi_{i}}{\partial t} \frac{\partial \Psi_{g-i}}{\partial t}
\end{aligned}
$$

Решая (1.33) с начальным условием $\Phi_{0}(0, t)=\Phi(0, t, 0)$, получаем описание производящей функции для модулярных деревьев $(g=0)$. Отметим, что пространство модулей $\bar{M}_{0, n}$ гладко и модулярные деревья не имеют автоморфизмов, так что для $g=0$ получаем настояшие многочлен Пуанкаре, или эйлерову характеристику, или число деревьев.

ТЕОРема 1.2. Формальнье ряды

$$
\alpha_{s}(t)=t-s \Phi_{0}(0, t)=t-s \sum_{n=3}^{\infty} v\left(M_{0, n}\right) \frac{t^{n-1}}{(n-1) !}
$$

$u$

$$
\beta_{s}(t)=t+s \Phi_{0}(s, t)=t+s \sum_{n=3}^{\infty}\left(\sum_{k=0}^{n-3} v\left(M_{0, n}^{k}\right) s^{k}\right) \frac{t^{n-1}}{(n-1) !}
$$

взаимообратны относительно операции композиции; функция $\Phi_{0}(s, t)$ удовлетворяет функииональным уравнениям

$$
\Phi_{0}(s, t)=\Phi_{0}\left(0, t+s \Phi_{0}(s, t)\right)
$$

$$
\Phi_{0}(0, t)=\Phi_{0}\left(s, t-s \Phi_{0}(0, t)\right)
$$

СЛЕДСТВИЕ 1.1. 1) Считающая функция для числа трехвалентных деревъев

$$
\Phi_{0}(s, t)=\frac{1-s t-\sqrt{1-2 s t}}{s^{2}}, \quad \Phi_{0}(1, t)=1-t-\sqrt{1-2 t}
$$

2) Считающая функиия для числа всех стабильньх деревьев $\Phi_{0}(s, t)$ удовлетворяет функичональному уравнению

$$
e^{t+s \Phi_{0}(s, t)}=1+t+(1+s) \Phi_{0}(s, t)
$$

и дифференциальному уравнению

$$
\left(\Phi_{0}(s, t)\right)_{t}^{\prime}=\frac{t+(s+1) \Phi_{0}(s, t)}{1-s\left[t+(s+1) \Phi_{0}(s, t)\right]} .
$$


3) Производящая функиия $\Phi_{0}(s, t)$ для многочлена Пуанкаре многообразия $\bar{M}_{0, n}$ удовлетворяет функциональному уравнению

$$
\left(1+t+s \Phi_{0}(s, t)\right)^{q}=q(q+s-1) \Phi_{0}(s, t)+q t+1
$$

и дифференциальному уравнению

$$
\left(\Phi_{0}(s, t)\right)^{\prime}=\frac{t+(q+s) \Phi_{0}(s, t)}{1+t-s t-s(q+s-1) \Phi_{0}(1, t)} .
$$

4) Производящая функиия $\Phi_{0}(s, t)$ для әйлеровой характеристики многообразия $\bar{M}_{0, n}$ удовлетворяет функциональному уравнению

$$
\ln \left(1+t+s \Phi_{0}(s, t)\right)=\frac{t+(s+1) \Phi_{0}(s, t)}{1+t+s \Phi_{0}(s, t)}
$$

и дифференциальному уравнению

$$
\left(\Phi_{0}(s, t)\right)_{t}^{\prime}=\frac{t+(s+1) \Phi_{0}(s, t)}{1+t-s t-s^{2} \Phi_{0}(s, t)} .
$$

Уравнения для многочлена Пуанкаре и эйлеровой характеристики хорошо известны для $s=1$ (см. [8]).

Функция $\Phi_{0}(s, t)$ сушественно используется в рекуррентных формулах для решения уравнений (1.35) для $g>0$, основанных на следующем интегральном представлении.

Teоpema 1.3. Решение

$$
\Psi(s, t, \hbar)=\sum_{g=0}^{\infty} \Psi_{g}(s, t) \hbar^{g-1}
$$

потенциального уравнения Бюргерса (1.7) с начальным условием $\Psi(0, t, \hbar)$ может бъть представлено в виде

$$
\begin{aligned}
& \Psi(s, t, \hbar)=\Psi\left(0, t+s \Phi_{0}(s, t), \hbar\right) \\
& +\frac{\hbar}{2} \int_{0}^{s}\left[\frac{\partial^{2} \Psi}{\partial t^{2}}+\left(\frac{\partial\left(\Psi-\Psi_{0}\right)}{\partial t}\right)^{2}\right]\left(\sigma, t+(s-\sigma) \Phi_{0}(s, t), \hbar\right) d \sigma .
\end{aligned}
$$

В результате мы имеем явные рекуррентные формулы.

СлЕДСТвиЕ 1.2. Для $g>0$

$$
\begin{aligned}
\Psi_{g}(s, t) & =\Psi_{g}\left(0, t+s \Phi_{0}(s, t)\right) \\
+ & \frac{1}{2} \int_{0}^{s}\left[\frac{\partial^{2} \Psi_{g-1}}{\partial t^{2}}+\sum_{i=1}^{g-1} \frac{\partial \Psi_{i}}{\partial t} \frac{\partial \Psi_{g-i}}{\partial t}\right]\left(\sigma, t+(s-\sigma) \Phi_{0}(s, t)\right) d \sigma .
\end{aligned}
$$

Теперь мы готовы применить (1.49) к любому случаю, для которого мы можем найти $\Phi_{0}(s, t)$ и начальное условие $\Psi(0, t, \hbar)$. 
1.3. Трехвалентные графы. Мы видели, что для трехвалентных графов $\Phi_{0}(s, t)=(1-s t-\sqrt{1-2 s t}) / s^{2}$ и $\Psi(0, t, \hbar)=t^{3} /(6 \hbar)$.

В этом случае можно найти однопараметрическое семейство явных решений уравнения (1.34), выраженных через модифицированные функции Бесселя $I_{\nu}(z)$ или функции Эйри $\operatorname{Ai}(z)$ и $\operatorname{Bi}(z)$ (определения см. в [9]). Все эти решения аналитичны вне прямой $s=0$, а на этой прямой они только бесконечно дифференцируемы, причем любое из решений дает одно и то же разложение в ряд по $s$ и $t$, который, впрочем, расходится.

Теорема 1.4. Считающая функиия $\Phi$ для трехвалентных графов

$$
\begin{aligned}
& \Phi(s, t, \hbar)=\sum_{g=0}^{\infty} \sum_{n=1}^{\infty} \sum_{k=0}^{3 g-3+n}\left[\sum_{\text {по трехвалентным графам } \Gamma} \frac{1}{|\mathrm{Aut} \Gamma|}\right] \frac{t^{n-1}}{(n-1) !} s^{k} \hbar^{g-1} \\
& \text { рода } g \text { с } k \text { ребрами } \\
& \text { и } n \text { полуребрами } \\
& =\frac{1-s t}{s^{2} \hbar}-\frac{\sqrt{1-2 s t}}{s^{2} \hbar}\left[\frac{C_{1} I_{-2 / 3}\left(\frac{(\sqrt{1-2 s t})^{3}}{3 s^{3} \hbar}\right)+C_{2} I_{2 / 3}\left(\frac{(\sqrt{1-2 s t})^{3}}{3 s^{3} \hbar}\right)}{C_{1} I_{1 / 3}\left(\frac{(\sqrt{1-2 s t})^{3}}{3 s^{3} \hbar}\right)+C_{2} I_{-1 / 3}\left(\frac{(\sqrt{1-2 s t})^{3}}{3 s^{3} \hbar}\right)}\right] \\
& =\frac{1-s t}{s^{2} \hbar}-\frac{2^{1 / 3}}{s \hbar^{2 / 3}}\left[\frac{C_{1}^{\prime} \operatorname{Ai}^{\prime}\left(\frac{1-2 s t}{2^{2 / 3} s^{2} \hbar^{2 / 3}}\right)+C_{2}^{\prime} \operatorname{Bi}^{\prime}\left(\frac{1-2 s t}{2^{2 / 3} s^{2} \hbar^{2 / 3}}\right)}{C_{1}^{\prime} \operatorname{Ai}\left(\frac{1-2 s t}{2^{2 / 3} s^{2} \hbar^{2 / 3}}\right)+C_{2}^{\prime} \operatorname{Bi}\left(\frac{1-2 s t}{2^{2 / 3} s^{2} \hbar^{2 / 3}}\right)}\right] \\
& =\frac{1-s t-\sqrt{1-2 s t}}{s^{2} \hbar}+\frac{s}{1-2 s t} W\left(\frac{s^{3} \hbar}{(\sqrt{1-2 s t})^{3}}\right) \text {, }
\end{aligned}
$$

где $C_{1}, C_{2}, C_{1}^{\prime}, C_{2}^{\prime}$ - произвольные константви $\left(C_{1}^{\prime}=\sqrt{3}\left(C_{2}-C_{1}\right)\right.$ и $C_{2}^{\prime}=$ $\left.C_{2}+C_{1}\right)$, а $W(u)$ - считающая функиия для трехвалентных графов с одним полуребром (см. также табличу 1 ):

$$
\begin{aligned}
W(u)= & \sum_{g=1}^{\infty}\left[\sum_{\begin{array}{c}
\text { по трехвалентным } \\
\text { гафам } \text { рода } g \\
\text { одним полуребром }
\end{array}} \frac{1}{|\mathrm{Aut} \Gamma|}\right] u^{g-1} \\
=\frac{1}{u} & {\left[1-\frac{C_{1} I_{-2 / 3}(1 /(3 u))+C_{2} I_{2 / 3}(1 /(3 u))}{C_{1} I_{1 / 3}(1 /(3 u))+C_{2} I_{-1 / 3}(1 /(3 u))}\right] } \\
=\frac{1}{u} & {\left[1-\left(1-\frac{(16 / 9-1)}{1 !} \frac{3}{8} u+\frac{(16 / 9-1)(16 / 9-9)}{2 !}\left(\frac{3}{8} u\right)^{2}\right.\right.} \\
& \left.-\frac{(16 / 9-1)(16 / 9-9)(16 / 9-25)}{3 !}\left(\frac{3}{8} u\right)^{3}+\cdots\right)^{3} \\
& \times\left(1-\frac{(4 / 9-1)}{1 !} \frac{3}{8} u+\frac{(4 / 9-1)(4 / 9-9)}{2 !}\left(\frac{3}{8} u\right)^{2}\right.
\end{aligned}
$$




$$
\begin{gathered}
\left.\left.-\frac{(4 / 9-1)(4 / 9-9)(4 / 9-25)}{3 !}\left(\frac{3}{8} u\right)^{3}+\cdots\right)^{-1}\right] \\
=\left(\sum_{n=0}^{\infty} \frac{(6 n+1) !}{2(2 n) !(3 n) !} \frac{u^{n}}{288^{n}}\right)\left(\sum_{n=0}^{\infty} \frac{(6 n) !}{(2 n) !(3 n) !} \frac{u^{n}}{288^{n}}\right)^{-1} \\
=\frac{1}{2}+\frac{5}{8} u+\frac{15}{8} u^{2}+\frac{1105}{128} u^{3}+\frac{1695}{32} u^{4}+\frac{414125}{1024} u^{5}+\cdots .
\end{gathered}
$$

Обозначим

$$
W(u)=\sum_{g=1}^{\infty} \tau_{g} u^{g-1} .
$$

Тогда ${ }^{1}$

$$
\sum_{\substack{\text { по трехвалентным графам } \\ \text { рода } g \text { с } k \text { ребрами } \\ \text { и } n \text { полуребрами }}} \frac{1}{|\operatorname{Aut} \Gamma|}= \begin{cases}(2 n-5) ! !, & g=0, n \geqslant 3 ; \\ \frac{1}{2}(2 n-2) ! !, & g=1, n \geqslant 1 ; \\ \tau_{g} \frac{(3 g+2 n-5) ! !}{(3 g-3) ! !}, & g \geqslant 2,\end{cases}
$$

причем числа $\tau_{g}$ при $g>1$ удовлетворяют следующим рекуррентным соотношениям:

$$
\tau_{g}=\frac{1}{2}\left((3 g-4) \tau_{g-1}+\sum_{i=1}^{g-1} \tau_{i} \tau_{g-i}\right) .
$$

СЛЕДСТВИЕ 1.3. Считающая функиия $\Psi$ для трехвалентных графов имеет вид:

$$
\begin{aligned}
\Psi(s, t, \hbar)= & \sum_{g=0}^{\infty} \sum_{n=0}^{\infty} \sum_{k=0}^{3 g-3+n}\left[\sum_{\begin{array}{c}
\text { по трехвалентным графам } \\
\text { рода } g \text { с } k \text { ребрами } \\
\text { и } n \text { полуребрами }
\end{array}} \frac{1}{|\mathrm{Aut} \Gamma|}\right] \frac{t^{n}}{n !} s^{k} \hbar^{g-1} \\
= & \frac{1}{6 s^{3} \hbar}\left[-2+6 s t-3 s^{2} t^{2}\right]-\frac{1}{6} \ln \left(s^{3} \hbar\right) \\
& \quad+\ln \left[C_{1}^{\prime} \operatorname{Ai}\left(\frac{1-2 s t}{2^{2 / 3} s^{2} \hbar^{2 / 3}}\right)+C_{2}^{\prime} \operatorname{Bi}\left(\frac{1-2 s t}{2^{2 / 3} s^{2} \hbar^{2 / 3}}\right)\right] \\
= & \frac{1}{6 s^{3} \hbar}\left[-2+6 s t-3 s^{2} t^{2}\right]-\frac{1}{2} \ln \left(s^{3} \hbar\right)+\frac{1}{2} \ln (1-2 s t)-\ln 3-\frac{1}{3} \ln 2 \\
& \quad+\ln \left[C_{1} I_{1 / 3}\left(\frac{(\sqrt{1-2 s t})^{3}}{3 s^{3} \hbar}\right)+C_{2} I_{-1 / 3}\left(\frac{(\sqrt{1-2 s t})^{3}}{3 s^{3} \hbar}\right)\right] \\
= & \frac{1}{6 s^{3} \hbar}\left[2(\sqrt{1-2 s t})^{3}-2+6 s t-3 s^{2} t^{2}\right] \\
& \quad-\frac{1}{4} \ln (1-2 s t)+V\left(\frac{s^{3} \hbar}{(\sqrt{1-2 s t})^{3}}\right)
\end{aligned}
$$

\footnotetext{
${ }^{1}$ Как заметил Дон Загир, правую часть формулы (1.53) можно единообразно записать в виде $\tau_{g}(3 g+2 n-5) ! ! /(3 g-3) ! !$ для всех значений $g$ и $n$ таких, что $n+2 g>2$, полагая $\tau_{1}=1 / 2$ и $\tau_{0}=-1$, поскольку естественно считать, что $0 ! !=1$ и $(-3) ! !=-1$.
} 
где $V(u)$ - считающая функция для трехвалентных графов без полуребер (см. также табличу 2): ${ }^{2}$

$$
\begin{aligned}
V(u)= & \sum_{g=2}^{\infty}\left[\sum_{\substack{\text { по трехвалентным } \\
\text { графам } \Gamma \text { рода } g}} \frac{1}{|\operatorname{Aut} \Gamma|}\right] u^{g-1} \\
= & \ln \left[\frac{C_{1} I_{1 / 3}(1 /(3 u))+C_{2} I_{-1 / 3}(1 /(3 u))}{3 \sqrt[3]{2} \sqrt{u}} e^{-1 /(3 u)]}\right. \\
= & \ln \left[1-\frac{(4 / 9-1)}{1 !} \frac{3}{8} u+\frac{(4 / 9-1)(4 / 9-9)}{2 !}\left(\frac{3}{8} u\right)^{2}\right. \\
& \left.\quad-\frac{(4 / 9-1)(4 / 9-9)(4 / 9-25)}{3 !}\left(\frac{3}{8} u\right)^{3}+\cdots\right] \\
= & \sum_{g=2}^{\infty} \frac{\tau_{g}}{3 g-3} u^{g-1}=\ln \left[\sum_{n=0}^{\infty} \frac{(6 n) !}{(2 n) !(3 n) !} \frac{u^{n}}{288^{n}}\right] \\
= & \frac{5}{24} u+\frac{5}{16} u^{2}+\frac{1105}{1152} u^{3}+\frac{565}{128} u^{4}+\frac{82825}{3072} u^{5}+\cdots,
\end{aligned}
$$

где $\tau_{g}, C_{i}$ и $C_{i}^{\prime}$ те же самье, что и в теореме 1.4 .

Одновременно мы построили однопараметрическое семейство решений задачи Коши для уравнения теплопроводности (1.27) с начальным условием $F(0, t)=$ $e^{t^{3} /(6 \hbar)}$ (неединственность решения задачи Коши для уравнения теплопроводности с быстро растущими начальными условиями (быстрее, чем $e^{t^{2}}$ ) была замечена А.Н. Тихоновым в 1935 г. [7]):

$$
\begin{aligned}
F(s, t)= & \sqrt{\frac{1-2 s t}{s^{3} \hbar}} e^{\frac{-2+6 s t-3 s^{2} t^{2}}{6 s^{3} \hbar}} \\
& \times\left[C_{1} I_{1 / 3}\left(\frac{(\sqrt{1-2 s t})^{3}}{3 s^{3} \hbar}\right)+C_{2} I_{-1 / 3}\left(\frac{(\sqrt{1-2 s t})^{3}}{3 s^{3} \hbar}\right)\right] \\
= & \frac{1}{\sqrt[4]{1-2 s t}} e^{\frac{1}{6 s^{3} \hbar}\left[2(\sqrt{1-2 s t})^{3}-2+6 s t-3 s^{2} t^{2}\right]} \\
& \times \frac{C_{1} I_{1 / 3}\left(\frac{(\sqrt{1-2 s t})^{3}}{3 s^{3} \hbar}\right)+C_{2} I_{-1 / 3}\left(\frac{(\sqrt{1-2 s t})^{3}}{3 s^{3} \hbar}\right)}{\sqrt{\frac{s^{3} \hbar}{(\sqrt{1-2 s t})^{3}}}} e^{-\frac{(\sqrt{1-2 s t})^{3}}{3 s^{3} \hbar}} .
\end{aligned}
$$

Второе представление в (1.57) дается, чтобы показать, что $F$ определена и бесконечно дифференцируема на действительной оси $s=0$ : это очевидно для первого сомножителя, а второй представляет собой $V\left(s^{3} \hbar /(\sqrt{1-2 s t})^{3}\right)$ (см. (1.56)).

\footnotetext{
${ }^{2}$ Дон Загир сообщил автору красивое комбинаторное рассуждение, сразу приводящее к формуле $V(u)=\ln \left[\sum_{n=0}^{\infty} \frac{(6 n) !}{(2 n) !(3 n) !} \frac{u^{n}}{288^{n}}\right]$.
} 
ТАБлицА 1. Первые два члена разложения $W(u)=\frac{1}{2}+\frac{5}{8} u+\cdots$

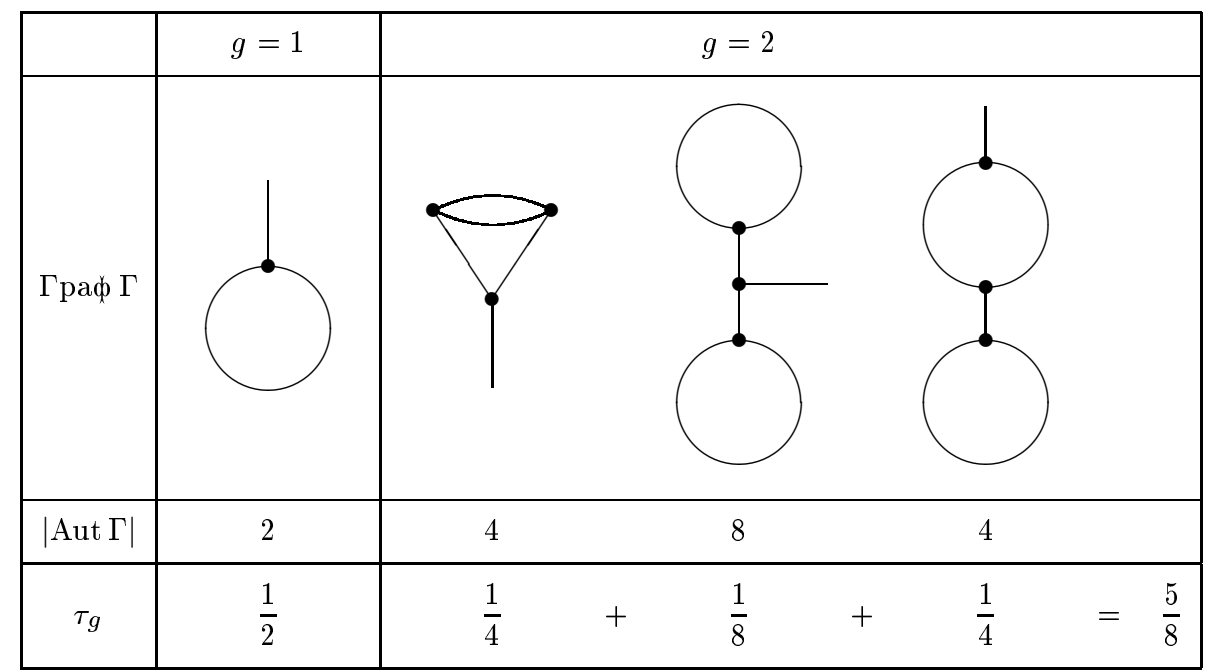

ТАБлицА 2. Первые два члена разложения $V(u)=\frac{5}{24} u+\frac{5}{16} u^{2}+\cdots$

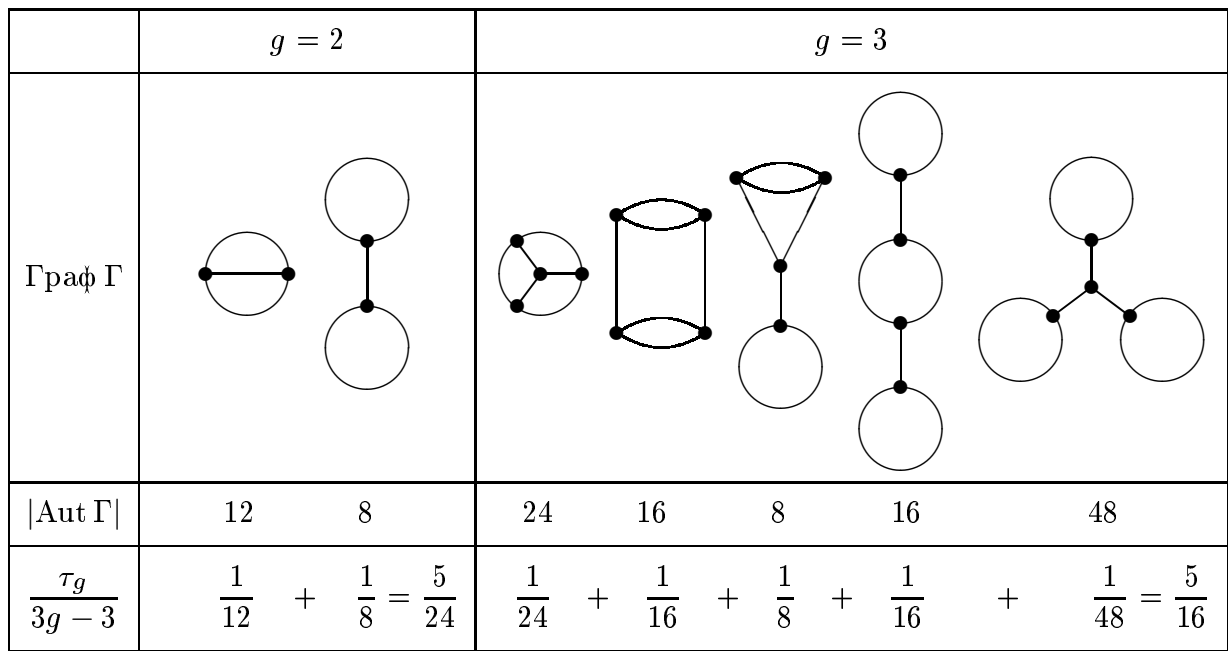

1.4. Считающая функция для числа всех комбинаторных графов. В этом случае функция $\Phi_{0}$ удовлетворяет функциональному уравнению (1.41) и дифференциальному уравнению (1.42). Начальное условие для уравнения Бюргерса (1.7) задается (1.15).

ТЕОРема 1.5. Члень $\Psi_{g}$ разложения сиитающей функиии

$$
\Psi(s, t, \hbar)=\sum_{g=0}^{\infty} \Psi_{g}(s, t) \hbar^{g-1}
$$


для числа всех (комбинаторных) графов выражаются следующим образом:

- npu $g=1$

$$
\Psi_{1}(s, t)=-\frac{1}{2} \ln \left(1-s\left(t+(s+1) \Phi_{0}(s, t)\right)\right) ;
$$

- npu $g>1$

$$
\Psi_{g}(s, t)=\frac{s^{g}\left(1+t+(s+1) \Phi_{0}(s, t)\right)}{\left(1-s\left(t+(s+1) \Phi_{0}(s, t)\right)\right)^{g}} P_{g}\left(\frac{s\left(1+t+(s+1) \Phi_{0}(s, t)\right)}{\left(1-s\left(t+(s+1) \Phi_{0}(s, t)\right)\right)}\right)
$$

где $P_{g}$ является многочленом степени $2 g-2$, удовлетворяющим следующему рекуррентному линейному дифференциальному уравнению: ${ }^{3}$

$$
\begin{aligned}
g P_{g}(u)+ & u P_{g}^{\prime}(u) \\
=\frac{1}{2}\left[u^{2}(u+1)^{2} P_{g-1}^{\prime \prime}(u)+u(u+1)[(2 g+1) u+3] P_{g-1}^{\prime}(u)\right. & \\
& +\left[\left(g^{2}-1\right) u^{2}-(3 g-2) u+1\right] P_{g-1}(u) \\
& +u \sum_{i=1}^{g-1}\left[u(u+1) P_{i}^{\prime}(u)+(i u+1) P_{i}(u)\right] \\
& \left.\times\left[u(u+1) P_{g-i}^{\prime}(u)+((g-i) u+1) P_{g-i}(u)\right]\right] .
\end{aligned}
$$

Ниже мы приводим первые три функции $\Psi_{g}$, вычисленные с помощью пакета MAPLE:

$$
\begin{aligned}
\Psi_{1}(s, t)= & \frac{1}{2} s t+\left(\frac{1}{4} s+\frac{1}{2} s^{2}\right) t^{2} \\
& +\left(\frac{1}{12} s+\frac{7}{12} s^{2}+\frac{2}{3} s^{3}\right) t^{3}+\left(\frac{1}{48} s+\frac{3}{8} s^{2}+\frac{59}{48} s^{3}+s^{4}\right) t^{4} \\
& +\left(\frac{1}{240} s+\frac{41}{240} s^{2}+\frac{19}{16} s^{3}+\frac{121}{48} s^{4}+\frac{8}{5} s^{5}\right) t^{5}+O\left(t^{6}\right), \\
\Psi_{2}(s, t)= & \left(\frac{1}{8} s^{2}+\frac{5}{24} s^{3}\right)+\left(\frac{1}{8} s^{2}+\frac{2}{3} s^{3}+\frac{5}{8} s^{4}\right) t \\
& +\left(\frac{1}{16} s^{2}+\frac{41}{48} s^{3}+\frac{109}{48} s^{4}+\frac{25}{16} s^{5}\right) t^{2} \\
& +\left(\frac{1}{48} s^{2}+\frac{47}{72} s^{3}+\frac{133}{36} s^{4}+\frac{53}{8} s^{5}+\frac{175}{48} s^{6}\right) t^{3} \\
& +\left(\frac{1}{192} s^{2}+\frac{203}{576} s^{3}+\frac{15}{4} s^{4}+\frac{1885}{144} s^{5}+\frac{3419}{192} s^{6}+\frac{525}{64} s^{7}\right) t^{4} \\
& +\left(\frac{1}{960} s^{2}+\frac{53}{360} s^{3}+\frac{7901}{2880} s^{4}+\frac{593}{36} s^{5}+\frac{7943}{192} s^{6}\right. \\
& \left.+\frac{1091}{24} s^{7}+\frac{1155}{64} s^{8}\right) t^{5}+O\left(t^{6}\right),
\end{aligned}
$$

\footnotetext{
${ }^{3}$ Следует формально положить $P_{1}(u)=(\ln (u+1)) /(2 u)$.
} 


$$
\begin{aligned}
\Psi_{3}(s, t)= & \left(\frac{1}{48} s^{3}+\frac{11}{48} s^{4}+\frac{25}{48} s^{5}+\frac{5}{16} s^{6}\right) \\
& +\left(\frac{1}{48} s^{3}+\frac{25}{48} s^{4}+\frac{119}{48} s^{5}+\frac{185}{48} s^{6}+\frac{15}{8} s^{7}\right) t \\
& +\left(\frac{1}{96} s^{3}+\frac{9}{16} s^{4}+\frac{241}{48} s^{5}+\frac{727}{48} s^{6}+\frac{1745}{96} s^{7}+\frac{15}{2} s^{8}\right) t^{2} \\
& +\left(\frac{1}{288} s^{3}+\frac{113}{288} s^{4}+\frac{295}{48} s^{5}+\frac{4595}{144} s^{6}+\frac{20357}{288} s^{7}+\frac{2225}{32} s^{8}+25 s^{9}\right) t^{3} \\
& +\left(\frac{1}{1152} s^{3}+\frac{29}{144} s^{4}+\frac{6101}{1152} s^{5}+\frac{6365}{144} s^{6}\right. \\
& \left.+\frac{184495}{1152} s^{7}+\frac{40465}{144} s^{8}+\frac{30075}{128} s^{9}+75 s^{10}\right) t^{4} \\
& +\left(\frac{1}{5760} s^{3}+\frac{157}{1920} s^{4}+\frac{20159}{5760} s^{5}+\frac{258589}{5760} s^{6}+\frac{31815}{128} s^{7}+\frac{794353}{1152} s^{8}\right. \\
& \left.+\frac{385291}{384} s^{9}+\frac{93555}{128} s^{10}+210 s^{11}\right) t^{5}+O\left(t^{6}\right) .
\end{aligned}
$$

1.5. Виртуальная эйлерова характеристика $\bar{M}_{g, n}$. В этом случае функция $\Phi_{0}$ удовлетворяет функциональному уравнению (1.45) и дифференциальному уравнению (1.46). Начальные условия для уравнения Бюргерса (1.7) или (1.8) задаются (1.24) или (1.25).

ТЕОРема 1.6. Члены $\Psi_{g}$ разложсения производящей функции

$$
\Psi(s, t, \hbar)=\sum_{g=0}^{\infty} \Psi_{g}(s, t) \hbar^{g-1}
$$

для виртуальной эйлеровой характеристики могут быть выражены следующим образом:

$$
\begin{aligned}
& - \text { при } g=1 \\
& \Psi_{1}(s, t)=-\frac{1}{2} \ln \left(1+t-s\left(t+s \Phi_{0}(s, t)\right)\right)+\frac{5}{12} \ln \left(1+t+s \Phi_{0}(s, t)\right) \\
& - \text { nри } g>1 \\
& \Psi_{g}(s, t)=\frac{1}{\left(1+t+s \Phi_{0}(s, t)\right)^{2 g-2}} \Psi_{g}\left(\frac{s\left(1+t+s \Phi_{0}(s, t)\right)}{1+t-s\left(t+s \Phi_{0}(s, t)\right)}, 0\right),
\end{aligned}
$$

$u \Psi_{g}(s, 0)$ является многочленом от $s$ степени $3 g-3$, удовлетворяющим следующим рекуррентным уравнениям:

$$
\begin{aligned}
\Psi_{g+1}(s, 0) & =\frac{B_{2 g}}{2 g(2 g+2)}+\frac{1}{2} \int_{0}^{s}\left[\sigma^{4} \frac{\partial^{2} \Psi_{g}}{\partial s^{2}}(\sigma, 0)+\sigma^{2}(3 \sigma+3-4 g) \frac{\partial \Psi_{g}}{\partial s}(\sigma, 0)\right. \\
- & 2(g-1)(\sigma-2 g+1) \Psi_{g}(\sigma, 0)+\sum_{i=1}^{g}\left(\sigma^{2} \frac{\partial \Psi_{i}}{\partial s}(\sigma, 0)-2(i-1) \Psi_{i}(\sigma, 0)\right) \\
& \left.\times\left(\sigma^{2} \frac{\partial \Psi_{g-i+1}}{\partial s}(\sigma, 0)-2(g-i) \Psi_{g-i+1}(\sigma, 0)\right)\right] d \sigma
\end{aligned}
$$


Старший коэффициент $\Psi_{g}(s, 0)$ равняется $\tau_{g} /(3 g-3)$ (определение $\tau_{g}$ см. в теореме 1.4).

В $\S 6$ мы приводим результаты расчетов по этим формулам, проведенных при помощи пакета MAPLE.

\section{§2. Склейки и разрезания модулярных графов}

Рассмотрим множество $\mathscr{G}_{g, n}^{k}$ стабильных модулярных графов рода $g$, имеюших $k$ ребер и $n$ полуребер. Для $k>0, n>0$ и $g \geqslant 0$ существует однозначно определенное отображение склейки

$$
\&: \mathscr{G}_{g-1, n+2}^{k-1} \rightarrow \mathscr{G}_{g, n}^{k}
$$

склеивающее первое и последнее полуребра модулярного графа $\Gamma \in \mathscr{G}_{g-1, n+2}^{k-1}$ в одно ребро $e^{\&}$ нового модулярного граффа $\&(\Gamma) \in \mathscr{G}_{g-1, n+2}^{k-1}$ (порядок остальных $n-2$ полуребер наследуется из Г). Отметим, что ребро $e^{\&}$ обладает однозначно определенной ориентацией (направленной от первого полуребра к последнему), так что мы построили отображение склейки

$$
\widetilde{\&}: \mathscr{G}_{g-1, n+2}^{k-1} \rightarrow \widetilde{\mathscr{G}}_{g, n}^{k}
$$

где $\widetilde{\mathscr{G}}_{g, n}^{k}-$ множество $\mathscr{G}_{g, n}^{k}$ стабильных модулярных графов рода $g$, имеюших $k$ ребер, $n$ полуребер и одно отмеченное ориентированное ребро. Отображение $\widetilde{\&}$ инъективно: граф̆ $Г$ может быть восстановлен из $\widetilde{\&}(\Gamma)$ разрезанием отмеченного ребра.

Теперь для $k>0, n \geqslant 0, g \geqslant 0$ возьмем некоторые неотрицательные целые числа $n_{1}, n_{2}, k_{1}, k_{2}, g_{1}, g_{2}$ такие, что $n_{1}+n_{2}=n, k_{1}+k_{2}=k-1$, $g_{1}+g_{2}=g$, и некоторое разбиение $\left(I_{1}, I_{2}\right)$ множества $\{1,2, \ldots, n\}=I_{1} \sqcup I_{2}$ такое, что $\left|I_{1}\right|=n_{1},\left|I_{2}\right|=n_{2}$. Пусть $I_{1}=\left\{i_{1}, i_{2}, \ldots, i_{n_{1}}\right\}$ и $I_{2}=\left\{j_{1}, j_{2}, \ldots, j_{n_{2}}\right\}$, где $i_{1}<i_{2}<\cdots<i_{n_{1}}$ и $j_{1}<j_{2}<\cdots<j_{n_{2}}$. Выберем два модулярных графа $\Gamma_{1} \in \mathscr{G}_{g_{1}, n_{1}+1}^{k_{1}}$ и $\Gamma_{2} \in \mathscr{G}_{g_{2}, n_{2}+1}^{k_{2}}$ и склеим первое полуребро модулярного графа $\Gamma_{1}$ и последнее полуребро модулярного графа $\Gamma_{2}$. Зададим упорядочение полуребер объединенного графа $\Gamma_{1} \& \Gamma_{2}$ следуюшим образом: $m$-е полуребро модулярного графа $\Gamma_{1}$ становится $i_{m-1}$-м полуребром модулярного графа $\Gamma_{1} \& \Gamma_{2}$ для $2 \leqslant m \leqslant n_{1}+1$ и $m$-е полуребро модулярного графа $\Gamma_{2}$ становится $j_{m}$-м полуребром модулярного графа $\Gamma_{1} \& \Gamma_{2}$ для $1 \leqslant m \leqslant n_{2}$. Таким образом, мы определили отображения склейки:

$$
\begin{aligned}
& \&: \mathscr{G}_{g_{1}, n_{1}+1}^{k_{1}} \times \mathscr{G}_{g_{2}, n_{2}+1}^{k_{2}} \times \mathscr{P}_{n_{1}, n_{2}} \rightarrow \mathscr{G}_{g, n}^{k}, \\
& \tilde{\&}: \mathscr{G}_{g_{1}, n_{1}+1}^{k_{1}} \times \mathscr{G}_{g_{2}, n_{2}+1}^{k_{2}} \times \mathscr{P}_{n_{1}, n_{2}} \rightarrow \widetilde{G}_{g, n}^{k},
\end{aligned}
$$

где $\mathscr{P}_{n_{1}, n_{2}}$ - множество всех разбиений. Повторяя приведенное вьше рассуждение, мы видим, что отображение (2.4) инъективно. Все отображения склей- 
ки $(2.1),(2.3)$ (и соответственно (2.2), (2.4)) можно объединить (при фиксированных $n$ и $g$ ) в одно отображение:

$$
\begin{aligned}
& \&_{g, n}: \mathscr{G}_{g-1, n+2}^{k-1} \cup \bigcup_{\substack{k_{1}+k_{2}=k-1 \\
g_{1}+g_{2}=g \\
n_{1}+n_{2}=n}}\left(\mathscr{G}_{g_{1}, n_{1}+1}^{k_{1}} \times \mathscr{G}_{g_{2}, n_{2}+1}^{k_{2}} \times \mathscr{P}_{n_{1}, n_{2}}\right) \rightarrow \mathscr{G}_{g, n}^{k} \\
& \left(\tilde{\&}_{g, n}: \mathscr{G}_{g-1, n+2}^{k-1} \cup \bigcup_{\substack{k_{1}+k_{2}=k-1 \\
g_{1}+g_{2}=g \\
n_{1}+n_{2}=n}}\left(\mathscr{G}_{g_{1}, n_{1}+1}^{k_{1}} \times \mathscr{G}_{g_{2}, n_{2}+1}^{k_{2}} \times \mathscr{P}_{n_{1}, n_{2}}\right) \rightarrow \widetilde{\mathscr{G}}_{g, n}^{k}\right) .
\end{aligned}
$$

Последнее отображение $\widetilde{\&}_{g, n}$, очевидно, биективно: для $(\vec{e}, \widetilde{\Gamma}) \in \widetilde{\mathscr{G}}_{g, n}^{k}$ обратное отображение $\widetilde{\&}_{g, n}^{-1}$ получается разрезанием отмеченного ориентированного ребра $\vec{e}$ графа $\widetilde{\Gamma} \in \mathscr{G}_{g, n}^{k}$ на два полуребра, причем упорядочение полуребер наследуется из графа $\widetilde{\Gamma}$. (Два новых разрезанных полуребра приобретают соответственно первый и последний номер согласно с ориентацией выделенного ребра.)

Рассмотрим проекцию

$$
\pi_{g, n}: \widetilde{\mathscr{G}}_{g, n}^{k} \rightarrow \mathscr{G}_{g, n}^{k}
$$

Выберем некоторый модулярный граф̆ $\widetilde{\Gamma} \in \mathscr{G}_{g, n}^{k} ;$ группа Aut $\widetilde{\Gamma}$ действует на множестве его ориентированных ребер $\vec{E}(\widetilde{\Gamma})$. Пары $(\vec{e}, \widetilde{\Gamma})$ из слоя $\pi_{g, n}^{-1}(\widetilde{\Gamma})$ находятся во взаимно однозначном соответствии с орбитами этого действия. Выберем представителей $\left\{\vec{e}_{\alpha}\right\}$ по одному из каждой орбиты, тогда

$$
2 k=|\vec{E}(\widetilde{\Gamma})|=\sum_{\vec{e}_{\alpha}}\left|\operatorname{Aut} \widetilde{\Gamma} \cdot \vec{e}_{\alpha}\right|=\sum_{\vec{e}_{\alpha}}\left(\operatorname{Aut} \widetilde{\Gamma}: \operatorname{Aut} \widetilde{\Gamma}_{\vec{e}_{\alpha}}\right)=\sum_{\vec{e}_{\alpha}} \frac{\operatorname{Aut} \widetilde{\Gamma}}{\operatorname{Aut} \widetilde{\Gamma}_{\vec{e}_{\alpha}}}
$$

где Aut $\widetilde{\Gamma}_{\vec{e}_{\alpha}}$ - стабилизатор ориентированного ребра $\vec{e}_{\alpha}$. Каждая пара $\left(e_{\alpha}, \widetilde{\Gamma}\right)$ лежит в однозначно определенном образе одного из отображений $\widetilde{\&}:(2.4)$, если ребро $e_{\alpha}$ разбивает модулярный граф $\widetilde{\Gamma}$ на две несвязные части, или $(2.2)$, если не разбивает. В первом случае

$$
\text { Aut } \widetilde{\Gamma}_{\vec{e}_{\alpha}} \cong \operatorname{Aut} \Gamma_{1} \times \operatorname{Aut} \Gamma_{2}
$$

во втором случае -

$$
\text { Aut } \widetilde{\Gamma}_{\vec{e}_{\alpha}} \cong \text { Aut } \Gamma \text {. }
$$

(В случае (2.9) $\widetilde{\Gamma}=\Gamma_{1} \& \Gamma_{2}$ и в случае $\left.(2.10) \widetilde{\Gamma}=\&(\Gamma).\right)$ Объединяя $(2.10),(2.9)$ и (2.8), получаем

$$
\frac{2 k}{|\operatorname{Aut} \widetilde{\Gamma}|}=\sum_{\Gamma \in \&_{g, n}^{-1}(\widetilde{\Gamma})} \frac{1}{|\operatorname{Aut} \Gamma|}+\sum_{\left(\Gamma_{1}, \Gamma_{2},\left(I_{1}, I_{2}\right)\right) \in \&_{g, n}^{-1}(\widetilde{\Gamma})} \frac{1}{\left|\operatorname{Aut} \Gamma_{1}\right|} \frac{1}{\left|\operatorname{Aut} \Gamma_{2}\right|}
$$


Пусть $\left\{\mu_{g, n}, 2(g-1)+n>0\right\}-$ множество (коммутативных) переменных. В (1.2) и (1.3) мы определили мономы

$$
\mu(\Gamma)=\frac{1}{|\operatorname{Aut} \Gamma|} \prod_{v \in V(\Gamma)} \mu_{g(v), \nu(v)}
$$

и многочлены

$$
\mu_{g, n}^{k}=\sum_{\Gamma \in \mathscr{G}_{g, n}^{k}} \mu(\Gamma) .
$$

Каждый из модулярных графов (или каждый из пар модулярных графов) в $\widetilde{\&}_{g, n}^{-1}(\widetilde{\Gamma})$ состоит из одного и того же набора вершин с теми же валентностями; поэтому, умножая (2.11) на $\prod_{v \in V(\widetilde{\Gamma})} \mu_{g(v), \nu(v)}$, получаем

$$
2 k \mu(\widetilde{\Gamma})=\sum_{\Gamma \in \tilde{\mathscr{E}}_{g, n}^{-1}(\widetilde{\Gamma})} \mu(\Gamma)+\sum_{\left(\Gamma_{1}, \Gamma_{2},\left(I_{1}, I_{2}\right)\right) \in \widetilde{\mathscr{E}}_{g, n}^{-1}(\widetilde{\Gamma})} \mu\left(\Gamma_{1}\right) \mu\left(\Gamma_{2}\right) .
$$

Суммируя (2.14) по всем $\widetilde{\Gamma} \in \mathscr{G}_{g, n}^{k}$, имеем

$$
2 k \mu_{g, n}^{k}=\mu_{g-1, n+2}^{k-1}+\sum_{\substack{k_{1}+k_{2}=k-1 \\
g_{1}+g_{2}=g \\
n_{1}+n_{2}=n}}\left(\begin{array}{c}
n \\
n_{1}
\end{array}\right) \mu_{g_{1}, n_{1}}^{k_{1}} \mu_{g_{2}, n_{2}}^{k_{2}} .
$$

Вспоминая определение производяшей функции (1.4)

$$
\Psi(s, t, \hbar)=\sum_{g=0}^{\infty} \sum_{n=0}^{\infty} \sum_{k=0}^{3 g-3+n} \mu_{g, n}^{k} \frac{t^{n}}{n !} s^{k} \hbar^{g-1}
$$

и умножая (2.15) на $t^{n} s^{k-1} \hbar^{g-1} /(2 n !)$, получаем потенциальное уравнение Бюргерса

$$
\frac{\partial \Psi}{\partial s}=\frac{\hbar}{2}\left[\frac{\partial^{2} \Psi}{\partial t^{2}}+\left(\frac{\partial \Psi}{\partial t}\right)^{2}\right]
$$

Теорема 1.1 доказана.

Используя подобные рассуждения, можно доказать формулу (1.18): для любой виртуальной мотивной меры $\widetilde{v}$

$$
\widetilde{v}\left(M_{\Gamma}\right)=\frac{1}{|\operatorname{Aut} \Gamma|} \prod_{v \in V(\Gamma)} \widetilde{v}\left(M_{g(v), \nu(v)}\right)
$$

Это очевидно, когда Г является деревом; любой модулярньй граф может быть построен из дерева последовательньми операциями склейки (2.1). Так что для доказательства нам нужно только сравнить $\widetilde{v}\left(M_{\Gamma}\right)$ и $\widetilde{v}\left(M_{\&(\Gamma)}\right)$ в $(2.1)$. Положим $(\vec{e}, \widetilde{\Gamma})=\widetilde{\&}(\Gamma)$ (это просто означает, что $\widetilde{\Gamma}=\&(\Gamma)$ и $\vec{e}$ является его выделенным 
ориентированњым ребром, полученным при склейке двух полуребер). Рассмотрим пространство модулей $\widetilde{M}_{\widetilde{\Gamma}}$, параметризуюшее стабильные пунктированные кривые Делиня-Мамффорда с одной отмеченной ветвью на одной из особых точек, двойственньй модулярный граф̆ которых совпадает с $\widetilde{\Gamma}$. Проекция $\pi: \widetilde{M}_{\widetilde{\Gamma}} \rightarrow M_{\widetilde{\Gamma}}-$ это $2 k$-листное неразветвленное накрытие орбиобразий; пространство $\widetilde{M}_{\widetilde{\Gamma}}$ распадается на несвязное объединение компонент, соответствующих орбитам действия группы Aut $\widetilde{\Gamma}$ на множестве ориентированных ребер $\vec{E}(\widetilde{\Gamma})$ модулярного графа $\widetilde{\Gamma}$. Обозначим компоненту, соответствующую орбите $\vec{e}$, через $\widetilde{M}_{\widetilde{\Gamma}, \vec{e}}$, тогда $\pi: \widetilde{M}_{\widetilde{\Gamma}, \vec{e}} \rightarrow$ $M_{\widetilde{\Gamma}}$ является неразветвленным накрытием орбиобразий степени $\left(\right.$ Aut $\widetilde{\Gamma}:$ Aut $\left.\widetilde{\Gamma}_{\vec{e}}\right)=$ $\mid$ Aut $\widetilde{\Gamma}|/|$ Aut $\widetilde{\Gamma}_{\vec{e}} \mid$. Поэтому

$$
\widetilde{v}\left(\widetilde{M}_{\widetilde{\Gamma}, \vec{e}}\right)=\frac{|\operatorname{Aut} \widetilde{\Gamma}|}{\left|\operatorname{Aut} \widetilde{\Gamma}_{\vec{e}}\right|} \widetilde{v}\left(\widetilde{M}_{\widetilde{\Gamma}}\right)
$$

Отображения склейки (2.1) и (2.2) определяют отображения склейки

$$
\begin{aligned}
& \&_{\Gamma}: M_{\Gamma} \rightarrow M_{\widetilde{\Gamma}}, \\
& \widetilde{\&}_{\Gamma}: M_{\Gamma} \rightarrow \widetilde{M}_{\widetilde{\Gamma}} .
\end{aligned}
$$

Образом $\widetilde{\&}_{\Gamma}$ является $\widetilde{M}_{\widetilde{\Gamma}, \vec{e}}$ и имеет место изоморфизм

$$
\widetilde{\&}_{\Gamma}: M_{\Gamma} \cong \widetilde{M}_{\widetilde{\Gamma}, \vec{e}}
$$

поэтому $\widetilde{v}\left(M_{\Gamma}\right)=\widetilde{v}\left(\widetilde{M}_{\widetilde{\Gamma}, \vec{e}}\right)$. Это завершает доказательство (1.18).

\section{§3. Решения уравнения Бюргерса}

В этом параграфе мы решаем уравнения Бюргерса (1.7) или (1.8) с помощью разложений (1.32) и (1.31):

$$
\begin{aligned}
& \Psi(s, t, \hbar)=\sum_{g=0}^{\infty} \Psi_{g}(s, t) \hbar^{g-1}, \\
& \Phi(s, t, \hbar)=\sum_{g=0}^{\infty} \Phi_{g}(s, t) \hbar^{g-1} .
\end{aligned}
$$

Подставляя в (1.7) и (1.8), мы получаем квазилинейное уравнение для $\Phi_{0}$

$$
\frac{\partial \Phi_{0}}{\partial s}=\Phi_{0} \frac{\partial \Phi_{0}}{\partial t}
$$

и рекуррентные квазилинейные уравнения для $\Phi_{g}$ и $\Psi_{g}$ для $g>0$

$$
\begin{aligned}
& \frac{\partial \Phi_{g}}{\partial s}=\frac{1}{2} \frac{\partial^{2} \Phi_{g-1}}{\partial t^{2}}+\Phi_{0} \frac{\partial \Phi_{g}}{\partial t}+\Phi_{g} \frac{\partial \Phi_{0}}{\partial t}+\sum_{i=1}^{g-1} \Phi_{i} \frac{\partial \Phi_{g-i}}{\partial t} \\
& \frac{\partial \Psi_{g}}{\partial s}=\frac{1}{2} \frac{\partial^{2} \Psi_{g-1}}{\partial t^{2}}+\Phi_{0} \frac{\partial \Psi_{g}}{\partial t}+\frac{1}{2} \sum_{i=1}^{g-1} \frac{\partial \Psi_{i}}{\partial t} \frac{\partial \Psi_{g-i}}{\partial t}
\end{aligned}
$$


Для $g=0$ имеется только квазилинейное уравнение (3.3). Уравнения для характеристик

$$
\frac{d s}{1}=-\frac{d t}{\Phi_{0}}=\frac{d \Phi_{0}}{0}
$$

Два первых интеграла (3.6)

$$
\Phi_{0}=C_{1}, \quad t+s \Phi_{0}=C_{2}
$$

Тогда общее решение (3.3) имеет вид

$$
f_{0}\left(\Phi_{0}, t+s \Phi_{0}\right)=0
$$

где $f_{0}$ - произвольная функция. Подставляя начальные условия $\Phi_{0}(0, t)$, получаем, что при $s=0 f_{0}\left(\Phi_{0}(0, t), t\right)=0$; это означает, что $f_{0}(a, b)=0$ равносильно $a=\Phi_{0}(0, b)$. Таким образом, уравнение $(3.8)$ дает функциональное уравнение для $\Phi_{0}(s, t)$

$$
\Phi_{0}(s, t)=\Phi_{0}\left(0, t+s \Phi_{0}(s, t)\right)
$$

Положим

$$
\alpha_{s}(t)=t-s \Phi_{0}(0, t), \quad \beta_{s}(t)=t+s \Phi_{0}(s, t) .
$$

Из (3.9) получаем, что $t+s \Phi_{0}(s, t)-s \Phi_{0}\left(0, t+s \Phi_{0}(s, t)\right)=t$, так что функция $\alpha_{s}$ является обратной к функции $\beta_{s}$ относительно композиции функций:

$$
\alpha_{s}\left(\beta_{s}(t)\right)=t, \quad \beta_{s}\left(\alpha_{s}(t)\right)=t .
$$

Таким образом, теорема 1.2 доказана.

Теперь рассмотрим квазилинейное уравнение (3.5). Уравнения для характеристик для (3.5):

$$
\frac{d s}{1}=-\frac{d t}{\Phi_{0}}=\frac{d \Psi_{g}}{\frac{1}{2} \frac{\partial^{2} \Psi_{g-1}}{\partial t^{2}}+\frac{1}{2} \sum_{i=1}^{g-1} \frac{\partial \Psi_{i}}{\partial t} \frac{\partial \Psi_{g-i}}{\partial t}} .
$$

Уравнения для характеристик для (3.4):

$$
\frac{d s}{1}=-\frac{d t}{\Phi_{0}}=\frac{d \Phi_{g}}{\Phi_{g} \frac{\partial \Phi_{0}}{\partial t}+\frac{1}{2} \frac{\partial^{2} \Phi_{g-1}}{\partial t^{2}}+\sum_{i=1}^{g-1} \Phi_{i} \frac{\partial \Phi_{g-i}}{\partial t}} .
$$

Обозначим знаменатель из (3.12) через $H_{g}(s, t)$ :

$$
H_{g}(s, t)=\frac{1}{2} \frac{\partial^{2} \Psi_{g-1}}{\partial t^{2}}+\frac{1}{2} \sum_{i=1}^{g-1} \frac{\partial \Psi_{i}}{\partial t} \frac{\partial \Psi_{g-i}}{\partial t} .
$$


Отметим, что $H_{g}$ зависит только от $\Psi_{i}$ при $i<g$. Мы уже нашли один первый интеграл для (3.13) и (3.12) при всех $g$ :

$$
\Phi_{0}(s, t)=C_{1} .
$$

Подставляя (3.15) в (3.9), мы видим, что

$$
\Phi_{0}\left(0, t+s C_{1}\right)=C_{1}
$$

Обозначим через $\varphi$ одну из ветвей обратной функции для $\Phi_{0}(0, t)$, тогда

$$
t=\varphi\left(C_{1}\right)-s C_{1} \text {. }
$$

Теперь другой первый интеграл для (3.5) может быть (рекуррентно) найден простьм интегрированием:

$$
\Psi_{g}-\int H_{g}\left(s, \varphi\left(C_{1}\right)-s C_{1}\right) d s=C_{2} .
$$

Исключая $C_{1}$, получаем

$$
\Psi_{g}-\Xi_{g}(s, t)=C_{2}
$$

где

$$
\Xi_{g}(s, t)=\int_{0}^{s} H_{g}\left(\sigma, t+(s-\sigma) \Phi_{0}(s, t)\right) d \sigma .
$$

Отметим, что мы выбираем константу интегрирования так, что $\Xi_{g}(0, t)=0$. Таким образом, общее решение (3.5) может быть записано в виде

$$
\Psi_{g}(s, t)=\Xi_{g}(s, t)+U_{g}\left(\Phi_{0}(s, t)\right)
$$

для произвольной функции $U_{g}$. Функция $U_{g}$ определяется тогда из начального условия:

$$
U_{g}\left(\Phi_{0}(0, t)\right)=\Psi_{g}(0, t) .
$$

Подставляя $t+s \Phi_{0}(s, t)$ вместо $t$ в $\Phi_{0}(0, t)$ и применяя формулу $(3.9)$, мы получаем рекуррентную формулу для решения уравнения Бюргерса:

$$
\begin{aligned}
\Psi_{g}(s, t)= & \Psi_{g}\left(0, t+s \Phi_{0}(s, t)\right) \\
& +\frac{1}{2} \int_{0}^{s}\left[\frac{\partial^{2} \Psi_{g-1}}{\partial t^{2}}+\sum_{i=1}^{g-1} \frac{\partial \Psi_{i}}{\partial t} \frac{\partial \Psi_{g-i}}{\partial t}\right]\left(\sigma, t+(s-\sigma) \Phi_{0}(s, t)\right) d \sigma .
\end{aligned}
$$

Теорема 1.3 доказана.

\section{§4. Случай $g=0$}

В этом параграфе мы при помощи теоремы 1.2 получаем единообразньм способом функциональные уравнения для $\Phi_{0}(s, t)$ во всех обсуждавшихся случаях. 
4.1. Считающие функции для числа трехвалентных деревьев. Для числа трехвалентных деревьев $\Phi_{0}(0, t)=t^{2} / 2$ (см. (1.12) для $d=3$ ). Чтобы найти обратную функцию для $\alpha_{s}(t)=t-s t^{2} / 2$, нужно только решить квадратное уравнение $\beta_{s}(t)-s \beta_{s}(t)^{2} / 2=t$. Его решение $\beta_{s}(t)=\alpha_{1}^{-1}(t)=(1-\sqrt{1-2 s t}) / s$ и, следовательно,

$$
\Phi_{0}(s, t)=\frac{1-s t-\sqrt{1-2 s t}}{s^{2}}, \quad \Phi_{0}(1, t)=1-t-\sqrt{1-2 t},
$$

- хорошо известная производящая функция для числа трехвалентных деревьев с отмеченньми полуребрами.

4.2. Считающие функции для числа всех стабильных деревьев. В этом случае (см. (1.15))

$$
\Phi_{0}(0, t)=e^{t}-t-1 .
$$

Подставляя в (1.38), получаем функциональное уравнение (1.41), из которого стандартно выводится дифференциальное уравнение (1.42).

4.3. Многочлен Пуанкаре и эйлерова характеристика многообразия $\bar{M}_{0, n}$. Многочлен Пуанкаре для $M_{0, n}$ от переменной $y$ совпадает с числом точек многообразия $M_{0, n}\left(\mathbb{F}_{q}\right)$ над конечным полем $\mathbb{F}_{q}\left(\right.$ с заменой $\left.q=y^{2}\right)$. На проективной прямой $\mathbb{P}^{1}\left(\mathbb{F}_{q}\right)$ всего $q+1$ точка; первые три можно действием проективной группы перевести в 0,1 и $\infty$; остальные $q-3$ точки можно выбрать

$$
(q-2)(q-3) \cdots(q-n+2)
$$

способами. Следовательно, производящая функция имеет вид:

$$
\Phi_{0}(0, t)=\sum_{n=3}^{\infty} \frac{(q-2) !}{(n-1) !(q-n+1) !} t^{n-1}=\frac{(1+t)^{q}-q t-1}{q(q-1)} .
$$

Подставляя в (1.38), получаем функциональное уравнение (1.43), из которого стандартно выводится дифференциальное уравнение (1.44).

Для случая эйлеровой характеристики достаточно просто положить $q=1$ в (4.4).

\section{§5. Считающая функция для трехвалентных графов}

В этом случае лучше начать с уравнения (1.8) для функции $\Phi(s, t, \hbar)$. Для любого трехвалентного графа рода $g$, имеюшего $k$ ребер и $n$ полуребер, $k=3 g-3+n$, поэтому $\Phi$ содержит только мономы

$$
s^{3 g-3+n} t^{n-1} \hbar^{g-1}=s\left(s^{3} \hbar\right)^{g}(s t)^{n-1}
$$

и, следовательно,

$$
\Phi(s, t, \hbar)=s Z\left(s^{3} \hbar, s t\right)
$$


для некоторой функции $Z(x, y)$. Подставляя $(5.2)$ в (1.8), получаем уравнение

$$
Z+3 x \frac{\partial Z}{\partial x}+y \frac{\partial Z}{\partial y}=\frac{x}{2} \frac{\partial^{2} Z}{\partial y^{2}}+x Z \frac{\partial Z}{\partial y} .
$$

Аналогично (1.31) получаем разложение

$$
Z(x, y)=\sum_{g=0}^{\infty} Z_{g}(y) x^{g-1}
$$

Тогда для $Z_{0}(y)$ имеем однородное уравнение

$$
-2 Z_{0}+y Z_{0}^{\prime}=Z_{0} Z_{0}^{\prime}
$$

Нужньм нам решением $(5.5)$ с начальньмм условием $Z_{0}(0)=0$ является

$$
Z_{0}(y)=1-y-\sqrt{1-2 y},
$$

которое, конечно, совпадает с (1.40). Для $g>0$ (5.3) дает следуюшее рекуррентное линейное уравнение для $Z_{g}(y)$, которое, конечно, эквивалентно (1.34):

$$
-2 Z_{g}+3 g Z_{g}+y Z_{g}^{\prime}=\frac{1}{2} Z_{g-1}^{\prime \prime}+Z_{0} Z_{g}^{\prime}+Z_{g} Z_{0}^{\prime}+\sum_{i=1}^{g-1} Z_{i}^{\prime} Z_{g-i}
$$

Нетрудно найти $Z_{1}$ и обшую форму для $Z_{g}$ (можно было использовать и $(1.49)$, что, правда, в этом случае привело бы к чуть более громоздким вычислениям):

$$
Z_{1}=\frac{1}{2(1-2 y)}, \quad Z_{g}=\frac{\tau_{g}}{(\sqrt{1-2 y})^{3 g-1}}
$$

для некоторых констант $\tau_{g}$ и $\tau_{1}=1 / 2$. Уравнение (5.7) дает рекуррентную формулу для $\tau_{g}, g>1$ :

$$
\tau_{g}=\frac{1}{3 g-2}\left(\frac{1}{2}(3 g-2)(3 g-4) \tau_{g-1}+\sum_{i=1}^{g-1}(3 i-1) \tau_{i} \tau_{g-i}\right),
$$

которую нетрудно представить в более удобном виде:

$$
\tau_{g}=\frac{1}{2}\left((3 g-4) \tau_{g-1}+\sum_{i=1}^{g-1} \tau_{i} \tau_{g-i}\right) .
$$

Вот первые четыре значения $\tau_{g}$ :

$$
\tau_{1}=\frac{1}{2}, \quad \tau_{2}=\frac{5}{8}, \quad \tau_{3}=\frac{15}{8}, \quad \tau_{4}=\frac{1105}{128} .
$$


Подставляя (5.8) в (5.4), получаем решение $Z$ в следуюшем виде:

$$
\begin{aligned}
Z & =Z_{0}+\sum_{g=1}^{\infty} \tau_{g} \frac{x^{g}}{(\sqrt{1-2 y})^{3 g-1}}=Z_{0}+\frac{x}{1-2 y} \sum_{g=1}^{\infty} \tau_{g}\left(\frac{x}{(\sqrt{1-2 y})^{3}}\right)^{3 g-1} \\
& =Z_{0}+\frac{x}{1-2 y} W\left(\frac{x}{(\sqrt{1-2 y})^{3}}\right)
\end{aligned}
$$

где $W$ - некоторая функция одной переменной. Подставляя (5.12) в (5.3), получаем обыкновенное дифференциальное уравнение для $W(u)$ :

$$
1+(8 u-2) W+\left(27 u^{2}-6 x\right) W^{\prime}+9 u^{3} W^{\prime \prime}+4 u W^{2}+6 u^{2} W^{\prime} W=0 .
$$

Умножая (5.13) на $u^{-2 / 3}$ и интегрируя, имеем

$$
-2 u^{1 / 3} W(u)+2 u^{4 / 3} W(u)+u^{4 / 3} W(u)^{2}+3 u^{7 / 3} W^{\prime}(u)+u^{1 / 3}+C=0 .
$$

Поскольку $W$ регулярно при $u=0, C=0$, разделив (5.14) на $u^{1 / 3}$, получим уравнение Риккати

$$
2(u-1) W(u)+u W(u)^{2}+1+3 u^{2} W^{\prime}(u)=0 .
$$

Для уравнения (5.15) $u=0$ является особой точкой, и оно имеет единственное решение в виде формального ряда

$$
W(u)=\frac{1}{2}+\frac{5}{8} u+\frac{15}{8} u^{2}+\frac{1105}{128} u^{3}+\frac{1695}{32} u^{4}+\frac{414125}{1024} u^{5}+O\left(u^{6}\right) .
$$

Общее решение уравнения (5.15) аналитически выражается через модифицированные функции Бесселя:

$$
W(u)=\frac{1}{u}\left(1-\frac{C_{1} I_{-2 / 3}(1 /(3 u))+C_{2} I_{2 / 3}(1 /(3 u))}{C_{1} I_{1 / 3}(1 /(3 u))+C_{2} I_{-1 / 3}(1 /(3 u))}\right)
$$

для любых $C_{1}, C_{2}$. С помощью асимптотических разложений модифицированных функций Бесселя (см. [9]) решение (при любых $C_{1}$ и $C_{2}$ ) можно представить как отношение двух степенных рядов:

$$
\begin{aligned}
W(u)= & \frac{1}{u}\left(1-\left(1-\frac{(16 / 9-1)}{1 !} \frac{3}{8} u+\frac{(16 / 9-1)(16 / 9-4)}{2 !}\left(\frac{3}{8} u\right)^{2}\right.\right. \\
& \left.-\frac{(16 / 9-1)(16 / 9-4)(16 / 9-25)}{3 !}\left(\frac{3}{8} u\right)^{3}+\cdots\right) \\
& \times\left(1-\frac{(4 / 9-1)}{1 !} \frac{3}{8} u+\frac{(4 / 9-1)(4 / 9-4)}{2 !}\left(\frac{3}{8} u\right)^{2}\right. \\
& \left.\left.-\frac{(4 / 9-1)(4 / 9-4)(4 / 9-25)}{3 !}\left(\frac{3}{8} u\right)^{3}+\cdots\right)^{-1}\right) .
\end{aligned}
$$


Теперь можно выписать ответ:

$$
\begin{aligned}
\Phi(s, t, \hbar) & =\frac{1}{s^{2} \hbar}[1-s t-\sqrt{1-2 s t}]+\frac{1}{s^{2}} \sum_{g=1}^{\infty} \tau_{g} \frac{s^{3 g} \hbar^{g-1}}{(\sqrt{1-2 s t})^{3 g-1}} \\
& =\frac{1-s t-\sqrt{1-2 s t}}{s^{2} \hbar}+\frac{s}{1-2 s t} \sum_{g=1}^{\infty} \tau_{g}\left(\frac{s^{3} \hbar}{(\sqrt{1-2 s t})^{3}}\right)^{g-1} \\
& =\frac{1-s t-\sqrt{1-2 s t}}{s^{2} \hbar}+\frac{s}{1-2 s t} W\left(\frac{s^{3} \hbar}{(\sqrt{1-2 s t})^{3}}\right) .
\end{aligned}
$$

Полезно также выразить решение $\Phi(s, t, \hbar)$ через функции Эйри (см. [9]):

$$
\begin{aligned}
\Phi(s, t, \hbar)= & \frac{1-s t}{s^{2} \hbar}-\frac{2^{1 / 3}}{s \hbar^{2 / 3}}\left(C_{1}^{\prime} \mathrm{Ai}^{\prime}\left(\frac{1-2 s t}{2^{2 / 3} s^{2} \hbar^{2 / 3}}\right)+C_{2}^{\prime} \mathrm{Bi}^{\prime}\left(\frac{1-2 s t}{2^{2 / 3} s^{2} \hbar^{2 / 3}}\right)\right) \\
& \times\left(C_{1}^{\prime} \operatorname{Ai}\left(\frac{1-2 s t}{2^{2 / 3} s^{2} \hbar^{2 / 3}}\right)+C_{2}^{\prime} \operatorname{Bi}\left(\frac{1-2 s t}{2^{2 / 3} s^{2} \hbar^{2 / 3}}\right)\right)^{-1}
\end{aligned}
$$

где $C_{1}^{\prime}=\sqrt{3}\left(C_{2}-C_{1}\right)$ и $C_{2}^{\prime}=C_{2}+C_{1}$. Теперь легко проинтегрировать и получить аналитическое выражение (1.55) для функции $\Psi(s, t, \hbar)$; константа интегрирования (зависяшая от $s$ и $\hbar$ ) может быть найдена из уравнения Бюргерса (1.7).

Теорема 1.4 и следствие 1.3 доказаны.

\section{§6. Виртуальная эйлерова характеристика $\bar{M}_{g, n}$}

Основной шаг в доказательстве теоремы 1.6 - заметить, что решения $\Psi_{g}(s, t)$ можно представить в виде

$$
\Psi_{g}(s, t)=\frac{1}{\left(1+t+s \Phi_{0}(s, t)\right)^{2 g-2}} P_{g}\left(\frac{s\left(1+t+s \Phi_{0}(s, t)\right)}{1+t-s\left(t+s \Phi_{0}(s, t)\right)}\right),
$$

где $P_{g}$ - некоторый многочлен. Для этого достаточно вычислить $\Psi_{g}(s, t)$ по формулам (1.49) для первых нескольких значений $g$, а затем доказать это утверждение индукцией по $g$, пользуясь уравнением (1.33). (При вьчислении производных функции $\Phi_{0}$ надо воспользоваться уравнением (1.46).) После этого остается только заметить, что при $t=0$ равенство (6.1) преврашается в $\Psi_{g}(s, 0)=P_{g}(s)$. Для вычисления старших коэффициентов уравнение (1.68) дает в точности рекуррентное соотношение (1.54), определяюшее числа $\tau_{g}$. Впрочем, это совпадение имеет чисто геометрическую природу: нетрудно видеть, что старшие коэффициенты многочленов $P_{g}(s)$ - это в точности коэффициенты при $s^{3 g-3} \hbar^{g-1}$ в разложении $\Psi_{g}(s, t)$ в ряд по $s, t, \hbar$, которые соответствуют вкладу в эйлерову характеристику $\bar{M}_{g, n}$ нульмерного страта $M_{g, n}^{3 g-3}$, соответствуюшего дискретному множеству максимально вырожденных кривых, двойственный граф которых является комбинаторным трехвалентным графом (1l-кривые в терминологии книги А.Н. Тюрина [10]).

Ниже мы приводим некоторые результаты вычислений по формулам (1.67), (1.68), выполненные в пакете MAPLE. 
Многочлены $\Psi_{g}(s, 0)$ для $g=2,3,4,5,6$ :

$$
\begin{aligned}
\Psi_{2}(s, 0)= & \frac{5}{24} s^{3}-\frac{1}{6} s^{2}+\frac{13}{288} s-\frac{1}{240} \\
\Psi_{3}(s, 0)= & \frac{5}{16} s^{6}-\frac{55}{96} s^{5}+\frac{35}{72} s^{4}-\frac{2539}{10368} s^{3}+\frac{1307}{17280} s^{2}-\frac{19}{1440} s+\frac{1}{1008}, \\
\Psi_{4}(s, 0)= & \frac{1105}{1152} s^{9}-\frac{1045}{384} s^{8}+\frac{8549}{2304} s^{7}-\frac{66773}{20736} s^{6}+\frac{182341}{92160} s^{5}-\frac{2235257}{2488320} s^{4} \\
& +\frac{187051}{622080} s^{3}-\frac{17063}{241920} s^{2}+\frac{6221}{604800} s-\frac{1}{1440} \\
\Psi_{5}(s, 0)= & \frac{565}{128} s^{12}-\frac{26015}{1536} s^{11}+\frac{145883}{4608} s^{10}-\frac{3182161}{82944} s^{9} \\
& +\frac{2805265}{82944} s^{8}-\frac{229328099}{9953280} s^{7}+\frac{374564131}{29859840} s^{6}-\frac{578872613}{104509440} s^{5} \\
& +\frac{114641981}{58060800} s^{4}-\frac{667199}{1209600} s^{3}+\frac{32821}{290304} s^{2}-\frac{181}{12096} s+\frac{1}{1056} \\
\Psi_{6}(s, 0)= & \frac{82825}{3072} s^{15}-\frac{400565}{3072} s^{14}+\frac{1266935}{4096} s^{13} \\
& -\frac{159107029}{331776} s^{12}+\frac{241682111}{442368} s^{11}-\frac{9702562787}{19906560} s^{10} \\
& +\frac{253843871663}{716636160} s^{9}-\frac{1079372228279}{5016453120} s^{8}+\frac{835339878797}{7524679680} s^{7} \\
& -\frac{614429790997}{12541132800} s^{6}+\frac{6419764103}{348364800} s^{5}-\frac{3031168109}{522547200} s^{4} \\
& +\frac{106613887}{72576000} s^{3}-\frac{24719227}{88704000} s^{2}+\frac{441541}{12700800} s-\frac{691}{327600}
\end{aligned}
$$

Начальные члены разложения функций $\Psi_{g}(s, t)$ для $g=1,2,3$ :

$$
\begin{aligned}
\Psi_{1}(s, t)= & \left(\frac{1}{2} s-\frac{1}{12}\right) t+\left(\frac{1}{2} s^{2}-\frac{7}{24} s+\frac{1}{24}\right) t^{2} \\
& +\left(\frac{2}{3} s^{3}-\frac{5}{8} s^{2}+\frac{2}{9} s-\frac{1}{36}\right) t^{3}+\left(s^{4}-\frac{41}{32} s^{3}+\frac{199}{288} s^{2}-\frac{3}{16} s+\frac{1}{48}\right) t^{4} \\
& +\left(\frac{8}{5} s^{5}-\frac{83}{32} s^{4}+\frac{89}{48} s^{3}-\frac{533}{720} s^{2}+\frac{1}{6} s-\frac{1}{60}\right) t^{5}+O\left(t^{6}\right), \\
\Psi_{2}(s, t)= & \left(\frac{5}{24} s^{3}-\frac{1}{6} s^{2}+\frac{13}{288} s-\frac{1}{240}\right) \\
& +\left(\frac{5}{8} s^{4}-\frac{3}{4} s^{3}+\frac{109}{288} s^{2}-\frac{13}{144} s+\frac{1}{120}\right) t \\
& +\left(\frac{25}{16} s^{5}-\frac{39}{16} s^{4}+\frac{325}{192} s^{3}-\frac{379}{576} s^{2}+\frac{67}{480} s-\frac{1}{80}\right) t^{2} \\
& +\left(\frac{175}{48} s^{6}-\frac{167}{24} s^{5}+\frac{3497}{576} s^{4}-\frac{677}{216} s^{3}+\frac{4393}{4320} s^{2}-\frac{7}{36} s+\frac{1}{60}\right) t^{3} \\
& +\left(\frac{525}{64} s^{7}-\frac{3547}{192} s^{6}+\frac{44519}{2304} s^{5}-\frac{9439}{768} s^{4}\right. \\
& \left.+\frac{17933}{3456} s^{3}-\frac{5065}{3456} s^{2}+\frac{23}{90} s-\frac{1}{48}\right) t^{4}
\end{aligned}
$$




$$
\begin{aligned}
& +\left(\frac{1155}{64} s^{8}-\frac{1123}{24} s^{7}+\frac{14579}{256} s^{6}-\frac{5485}{128} s^{5}\right. \\
& \left.+\frac{11887}{540} s^{4}-\frac{3833}{480} s^{3}+\frac{5801}{2880} s^{2}-\frac{97}{300} s+\frac{1}{40}\right) t^{5}+O\left(t^{6}\right), \\
& \Psi_{3}(s, t)=\left(\frac{5}{16} s^{6}-\frac{55}{96} s^{5}+\frac{35}{72} s^{4}-\frac{2539}{10368} s^{3}+\frac{1307}{17280} s^{2}-\frac{19}{1440} s+\frac{1}{1008}\right) \\
& +\left(\frac{15}{8} s^{7}-\frac{395}{96} s^{6}+\frac{305}{72} s^{5}-\frac{9259}{3456} s^{4}\right. \\
& \left.+\frac{29311}{25920} s^{3}-\frac{341}{1080} s^{2}+\frac{19}{360} s-\frac{1}{252}\right) t \\
& +\left(\frac{15}{2} s^{8}-\frac{3665}{192} s^{7}+\frac{4415}{192} s^{6}-\frac{119495}{6912} s^{5}\right. \\
& \left.+\frac{928913}{103680} s^{4}-\frac{171107}{51840} s^{3}+\frac{1819}{2160} s^{2}-\frac{15}{112} s+\frac{5}{504}\right) t^{2} \\
& +\left(25 s^{9}-\frac{4625}{64} s^{8}+\frac{28655}{288} s^{7}-\frac{1790105}{20736} s^{6}+\frac{1360669}{25920} s^{5}\right. \\
& \left.-\frac{1821137}{77760} s^{4}+\frac{66737}{8640} s^{3}-\frac{27491}{15120} s^{2}+\frac{415}{1512} s-\frac{5}{252}\right) t^{3} \\
& +\left(75 s^{10}-\frac{62075}{256} s^{9}+\frac{864475}{2304} s^{8}-\frac{1131595}{3072} s^{7}+\frac{35398361}{138240} s^{6}\right. \\
& \left.-\frac{5165251}{38880} s^{5}+\frac{16351757}{311040} s^{4}-\frac{141479}{8960} s^{3}+\frac{69679}{20160} s^{2}-\frac{2995}{6048} s+\frac{5}{144}\right) t^{4} \\
& +\left(210 s^{11}-\frac{192115}{256} s^{10}+\frac{247385}{192} s^{9}-\frac{13001167}{9216} s^{8}+\frac{76135781}{69120} s^{7}\right. \\
& -\frac{3737291}{5760} s^{6}+\frac{11534753}{38880} s^{5}-\frac{165193453}{1555200} s^{4}+\frac{17647}{600} s^{3}-\frac{911023}{151200} s^{2} \\
& \left.+\frac{155}{189} s-\frac{1}{18}\right) t^{5}+O\left(t^{6}\right) \text {. }
\end{aligned}
$$

Таблицы значений $\widetilde{\chi}\left(\bar{M}_{g, 0}\right)$ и $\widetilde{\chi}\left(\bar{M}_{g, 1}\right), g \leqslant 20$. Нетрудно заметить, что в этом интервале значений $g$ эйлерова характеристика $\widetilde{\chi}\left(\bar{M}_{g, 0}\right)$ растет примерно как $C(g-1) ! / 2^{g-1}$, а отношение

$$
\frac{\widetilde{\chi}\left(\bar{M}_{g, 1}\right)}{\widetilde{\chi}\left(\bar{M}_{g, 0}\right)(2 g-2)}
$$

растет от 1.025 для $g=3$ до 1.038 для $g=20$. 


\begin{tabular}{|c|c|}
\hline$g$ & $\tilde{\chi}\left(\bar{M}_{g, 0}\right)$ \\
\hline 2 & $\frac{119}{1440} \approx 0.0826$ \\
\hline 3 & $\frac{8027}{181440} \approx 0.0442$ \\
\hline 4 & $\frac{2097827}{43545600} \approx 0.0482$ \\
\hline 5 & $\frac{150427667}{1916006400} \approx 0.0785$ \\
\hline 6 & $\frac{31966432414753}{188305108992000} \approx 0.170$ \\
\hline 7 & $\frac{21067150021261}{46115536896000} \approx 0.457$ \\
\hline 8 & $\frac{27108194937436478387}{18438836272496640000} \approx 1.470168428$ \\
\hline 9 & $\frac{12253091020103495716943}{2225676001833123840000} \approx 5.505334564$ \\
\hline 10 & $\frac{41107639746528672580958364833}{1748045931839735463936000000} \approx 23.51633844$ \\
\hline 11 & $\frac{18149470500315527186930400759373}{160820225729255662682112000000} \approx 112.8556462$ \\
\hline 12 & $\frac{19004221040884074685037446900552041691}{31610823569342493056795934720000000} \approx 601.1934804$ \\
\hline 13 & $\frac{1335395944593790109991624206528868880873}{379329882832109916681551216640000000} \approx 3520.407975$ \\
\hline 14 & $\frac{2697359250099761465877837488047416054790459}{120006181114158410004708930355200000000} \approx 22476.83599$ \\
\hline 15 & $\frac{17628737527982037548325073368636345668379043678957}{113436082710520465373771125836113510400000000} \approx 155406.7904$ \\
\hline 16 & $\frac{61187507009333322043736181893289455692441208195878609}{52893624852448399854284136389867785420800000000} \approx 1156803.059$ \\
\hline 17 & $\frac{71372306743070002809491037076029984614872395664643491}{7737834356680016697596425386187554816000000000} \approx 9223809.073$ \\
\hline 18 & $\begin{array}{r}\frac{17198235432952170987858390769814893434655150721674671445771265141}{219267898302032160155302114911031967019769528320000000000} \\
\approx 78434807.68\end{array}$ \\
\hline 19 & $\begin{array}{r}\frac{13050435425469643163551878925079739017685769865160451968198706727723}{18418503457370701453045377652526685229660640378880000000000} \\
\approx 708550260.6\end{array}$ \\
\hline 20 & $\begin{array}{r}137014760506364785741048203429669320537974177259444567259217133497233731 \\
20219257750768810601193019037621586300795818606592000000000000 \\
\approx 6776448582.0\end{array}$ \\
\hline
\end{tabular}




\begin{tabular}{|c|c|}
\hline$g$ & $\tilde{\chi}\left(\bar{M}_{g, 1}\right)$ \\
\hline 1 & $\frac{5}{12} \approx 0.4166666667$ \\
\hline 2 & $\frac{247}{1440} \approx 0.1715277778$ \\
\hline 3 & $\frac{13159}{72576} \approx 0.1813133818$ \\
\hline 4 & $\frac{5160601}{17418240} \approx 0.2962756857$ \\
\hline 5 & $\frac{1060344499}{1642291200} \approx 0.6456495042$ \\
\hline 6 & $\frac{43927799939987}{25107347865600} \approx 1.749599367$ \\
\hline 7 & $\frac{25578458051299001}{4519322615808000} \approx 5.659799095$ \\
\hline 8 & $\frac{71323310082487963309}{3352515685908480000} \approx 21.27456417$ \\
\hline 9 & $\frac{48270890814008387585027269}{529710888436283473920000} \approx 91.12686159$ \\
\hline 10 & $\frac{1532013946846243955713315776917}{3496091863679470927872000000} \approx 438.2075777$ \\
\hline 11 & $\frac{2255889841768911901484548469527387}{964921354375533976092672000000} \approx 2337.900215$ \\
\hline 12 & $\frac{288832892614815185388417599064551131741}{21073882379561662037863956480000000} \approx 13705.72766$ \\
\hline 13 & $\frac{66447212654413192038655941663348291926069}{758659765664219833363102433280000000} \approx 87584.99615$ \\
\hline 14 & $\frac{123070096996308531323829981549308669630859857}{203087383423960386161815112908800000000} \approx 605995.7784$ \\
\hline 15 & $\frac{146281181967774383738497529449993443280442541690511}{32410309345862990106791750238889574400000000} \approx 4513415.173$ \\
\hline 16 & $\frac{133309147159236466453784033068792506720345957334028501807}{3702553739671387989799889547290744979456000000000} \approx 36004648.83$ \\
\hline 17 & $\frac{2721690926359201802650400830738540838572166621421649160557}{8886128975211331175519734913497787950694400000000} \approx 306285327.8$ \\
\hline 18 & $\begin{array}{l}\frac{123136066030368677688394485156439501180080883329398977909415435779}{44489138785919568727162747952963007801112657920000000000} \\
\approx 2767778145.0\end{array}$ \\
\hline 19 & $\begin{aligned} \begin{array}{l}975371306046856089312443646349848042163131041618448148820157981265999 \\
36837006914741402906090755305053370459321280757760000000000\end{array} \\
\approx 26478028150.0\end{aligned}$ \\
\hline 20 & $\begin{array}{r}183782438297282310449428294736692535953487484512586556016473804299555114127 \\
687454763526139560440562647279133934227057832624128000000000000 \\
\end{array}$ \\
\hline
\end{tabular}




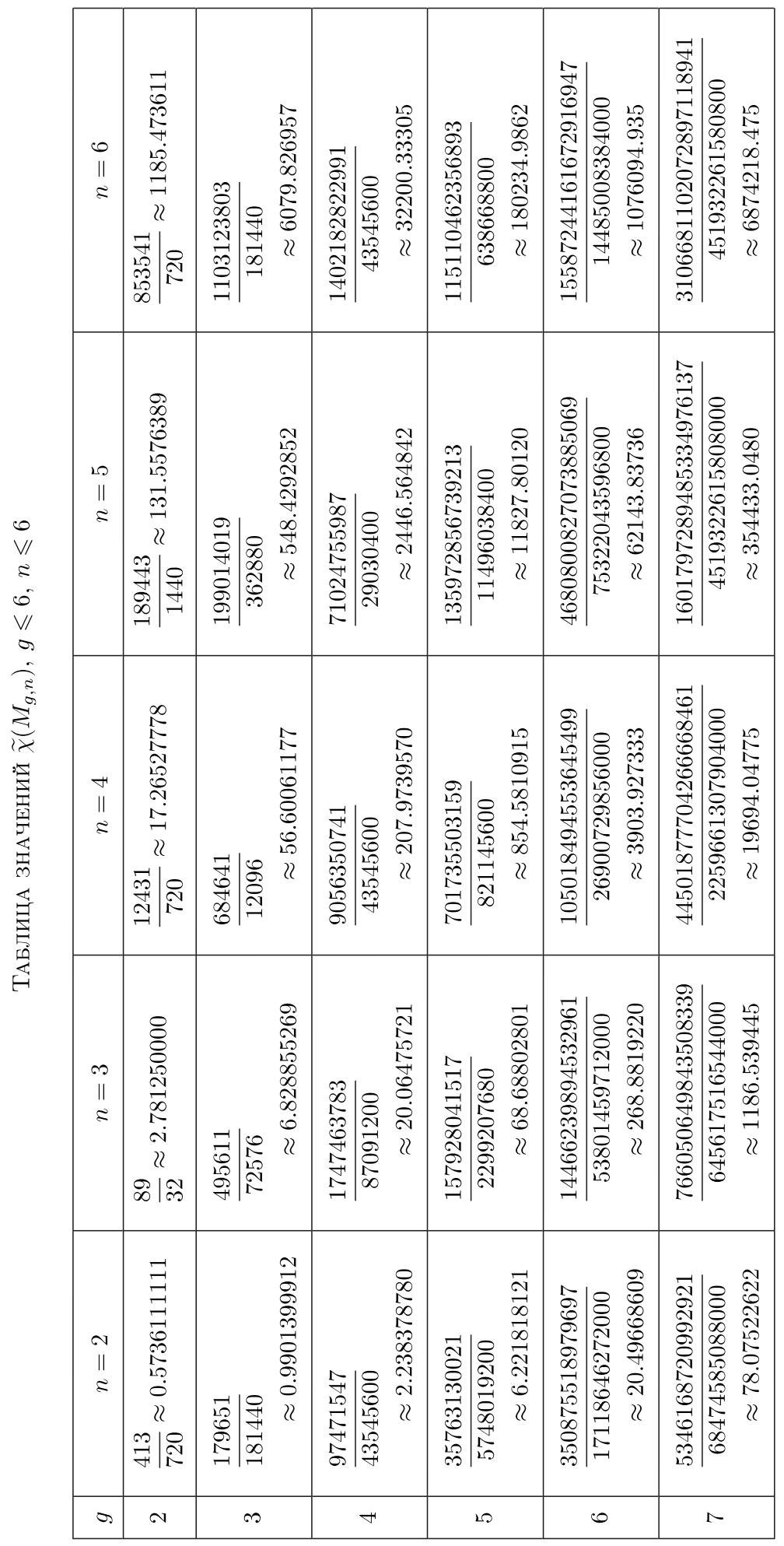


В заключение хотелось бы поблагодарить Г.Б. Шабата, обратившего внимание автора на производящие функции модулярных деревьев, и Ю.И. Манина за полезные обсуждения. Особенную благодарность автор хотел бы выразить Дону Загиру за целый ряд исправлений и упрощений в окончательном варианте текста. Также автор выражает признательность Институту Макса Планка в Бонне, чьим гостеприимством пользовался при завершении этой работы.

\section{Список литературы}

1. Fulton $W$., MacPherson $R$. A compactification of configuration spaces // Ann. of Math. (2). 1994. V. 139. P. 183-225.

2. Manin Yu. I. Generating functions in algebraic geometry and sums over trees // The moduli spaces of curves / ed. Dijkgraaf et al. Boston, MA: Birkhäuser Boston, 1995. P. 401-417. (Progr. Math. V. 129.)

3. Harer J., Zagier D. The Euler characteristic of the moduli space of curves // Invent. Math. 1986. V. 85. №3. P. 457-485.

4. Cole J. D. On a quasi-linear parabolic equation occurring in aerodynamics // Quart. App. Math. 1951. V. 9. P. 225-236.

5. Hopf E. The partial differential equation $u_{t}+u u_{x}=\mu u_{x x} / /$ Comm. Pure Appl. Math. 1950. V. 3. P. 201-230.

6. Getzler E. The semi-classical aproximation for modular operads // Comm. Math. Phys. 1998. V. 194. P. 481-492.

7. Tychonoff $A$. Théorèmes d'unicitépour l'équation de la chaleur // Матем. сб. 1935. Т. 42. № 2. C. $199-216$.

8. Манин Ю. И. Фробениусовы многообразия, квантовые когомологии и пространства модулей. М.: Факториал Пресс, 2002.

9. Абрамовии М., Стиган И. Справочник по специальным функциям с формулами, графиками и математическими таблицами. М.: Наука, 1979.

10. Тюрин А.Н. Квантование, классическая и квантовая теория поля и тэта-функции. М.-Ижевск: Институт компюютерных исследований, 2003.

Московский государственньй институт радиотехники, электроники и автоматики (технический университет)

E-mail: artamkin@mail.ru
Поступила в редакцию 31.03.2005 